Agnieszka Poulain-Paul ${ }^{1,2}$, Ayoub Nassour ${ }^{1}$, Christian Jelsch ${ }^{1}$, Benoit Guillot ${ }^{1}$, Maciej Kubicki ${ }^{2}$, Claude Lecomte ${ }^{1 *}$

${ }^{1}$ CRM2 UMR CNRS and Lorraine University, BP 70239, 54506 Vandoeuvre-les-Nancy Cedex, France.

${ }^{2}$ Faculty of Chemistry, Adam Mickiewicz University, Grunwaldzka 6, 60-780 Poznań, Poland.

\title{
A critical analysis of dipole moment calculations as obtained from experimental and theoretical structure factors.
}


Constrains/restraints imposed on the symmetry and simmilarity of the equivalent atoms:

a) symmetry:

$\begin{array}{ll} & \text { constrained } \\ \text { atom } & \text { symemtry } \\ \text { C1 } & \text { mymz } \\ \text { C2 } & \text { mymz } \\ \text { C3 } & \text { mymz } \\ \text { C4 } & \text { mymz } \\ \text { C5 } & \text { mymz } \\ \text { C6 } & \text { mymz } \\ \text { C7 } & m z \\ \text { C8 } & m z \\ \text { C9 } & m z \\ \text { C71 } & 3 m \\ \text { C91 } & c y \\ \text { N1 } & m y m z \\ \text { N2 } & m y m z \\ \text { N8 } & m y m z \\ \text { N91 } & c y \\ \text { O81 } & m z \\ \text { O82 } & m z\end{array}$

where $\mathrm{m}$ - mirror plane, $3 \mathrm{~m}$ - threefold axe, cy - cyllindrical symmetry.

b) equivalent atoms for similarity constraints/restraints on Plm, Pval, $\kappa, \kappa^{\prime}$ :

$\mathrm{C} 2$ and $\mathrm{C} 6$

$\mathrm{C} 3$ and $\mathrm{C} 5$

$\mathrm{O} 81$ and $\mathrm{O} 82$

$\mathrm{H} 2$ and $\mathrm{H} 6$

$\mathrm{H} 3$ and $\mathrm{H} 5$

$\mathrm{H} 71, \mathrm{H} 72$ and $\mathrm{H} 73$ 
Table S1. MM of theoretical data with scale factor refined to reduce the effect of different wave functions.

\begin{tabular}{|c|c|c|c|c|c|c|c|c|c|}
\hline Model & $\begin{array}{l}\text { Multipolar } \\
\text { expansion level } \\
\text { of non-hydrogen } \\
\text { / hydrogen } \\
\text { atoms }\end{array}$ & $\begin{array}{l}\text { Treatment of } \\
\kappa \text { and } \kappa^{\prime} \text { of } \\
\text { hydrogen } \\
\text { atoms }\end{array}$ & $\begin{array}{l}\text { Parameter } \\
\text { refined in order } \\
\text { to remove the } \\
\text { negative electron } \\
\text { density }\end{array}$ & $\begin{array}{l}\text { No } \\
\text { reflections/ } \\
\text { No } \\
\text { parameters }\end{array}$ & $\begin{array}{l}\text { Restraints/ } \\
\text { Constraints }\end{array}$ & $\begin{array}{l}\mathrm{RF} \\
\text { factor } \\
w \mathrm{R} 2 \mathrm{~F} \\
\text { factor }\end{array}$ & Goof & $\begin{array}{l}\text { Dipole moment } \\
\text { value } \\
\text { (module charge } \\
\text { dipole/atomic } \\
\text { dipole) [D] }\end{array}$ & 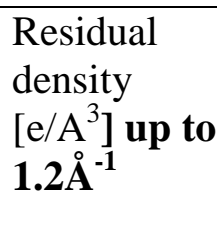 \\
\hline $1 \mathrm{~m}$ & HEX / QUA & refined & SCA & \begin{tabular}{ll|}
15074 & $/$ \\
532 & \\
\end{tabular} & - & $\begin{array}{l}0.548 \\
0.526\end{array}$ & 0.064 & $\begin{array}{l}14.85 \\
(13.82 / 2.29)\end{array}$ & $\begin{array}{l}0.287(15) / \\
-0.260(15)\end{array}$ \\
\hline 1n & HEX / QUA & 1.16 & SCA & \begin{tabular}{ll|}
15074 & $/$ \\
532 &
\end{tabular} & - & $\begin{array}{l}0.554 \\
0.531\end{array}$ & 0.064 & $\begin{array}{l}15.32 \\
(14.49 / 2.12)\end{array}$ & $\begin{array}{l}0.291(15) / \\
-0.258(15)\end{array}$ \\
\hline 10 & OCT / DIP & refined & SCA & \begin{tabular}{ll|}
15074 & $/$ \\
339 & \\
\end{tabular} & - & $\begin{array}{l}0.629 \\
0.636\end{array}$ & 0.076 & $\begin{array}{l}12.92 \\
(12.94 / 1.45)\end{array}$ & $\begin{array}{l}0.287(18) / \\
-0.248(18)\end{array}$ \\
\hline $1 \mathrm{p}$ & OCT / DIP & 1.16 & SCA & \begin{tabular}{ll|}
15074 & $/$ \\
339 & \\
\end{tabular} & - & $\begin{array}{l}0.630 \\
0.646\end{array}$ & 0.078 & $\begin{array}{l}13.04 \\
(12.62 / 1.40)\end{array}$ & $\begin{array}{l}0.283(18) / \\
-0.252(18)\end{array}$ \\
\hline $1 q$ & HEX / QUA & refined & SCA & $\begin{array}{l}15074 \\
532\end{array}$ & $\begin{array}{l}\mathrm{R}_{\text {free }} \\
\text { restraints }\end{array}$ & $\begin{array}{l}0.560 \\
0.542\end{array}$ & 0.053 & $\begin{array}{l}15.17 \\
(13.63 / 2.39)\end{array}$ & $\begin{array}{l}0.288(16) / \\
-0.261(16)\end{array}$ \\
\hline $1 \mathrm{r}$ & HEX / QUA & 1.16 & SCA & $\begin{array}{l}15074 \\
532 \\
\end{array}$ & $\begin{array}{l}\mathrm{R}_{\text {free }} \\
\text { restraints }\end{array}$ & $\begin{array}{l}0.565 \\
0.546\end{array}$ & 0.060 & $\begin{array}{l}15.64 \\
(14.29 / 2.22)\end{array}$ & $\begin{array}{l}0.292(16) / \\
-0.258(16)\end{array}$ \\
\hline $1 \mathrm{~s}$ & OCT / DIP & refined & SCA & $\begin{array}{l}15074 \\
339\end{array}$ & $\begin{array}{l}\mathrm{R}_{\text {free }} \\
\text { restraints }\end{array}$ & $\begin{array}{l}0.638 \\
0.649\end{array}$ & 0.068 & $\begin{array}{l}13.50 \\
(12.96 / 1.32)\end{array}$ & $\begin{array}{l}0.287(18) / \\
-0.248(18)\end{array}$ \\
\hline $1 \mathrm{t}$ & OCT / DIP & 1.16 & SCA & \begin{tabular}{ll|}
15074 & $/$ \\
339 &
\end{tabular} & $\begin{array}{l}\mathrm{R}_{\text {free }} \\
\text { restraints }\end{array}$ & $\begin{array}{l}0.637 \\
0.660\end{array}$ & 0.076 & $\begin{array}{l}13.28 \\
(12.26 / 1.49)\end{array}$ & $\begin{array}{l}0.282(19) / \\
-0.252(19)\end{array}$ \\
\hline
\end{tabular}




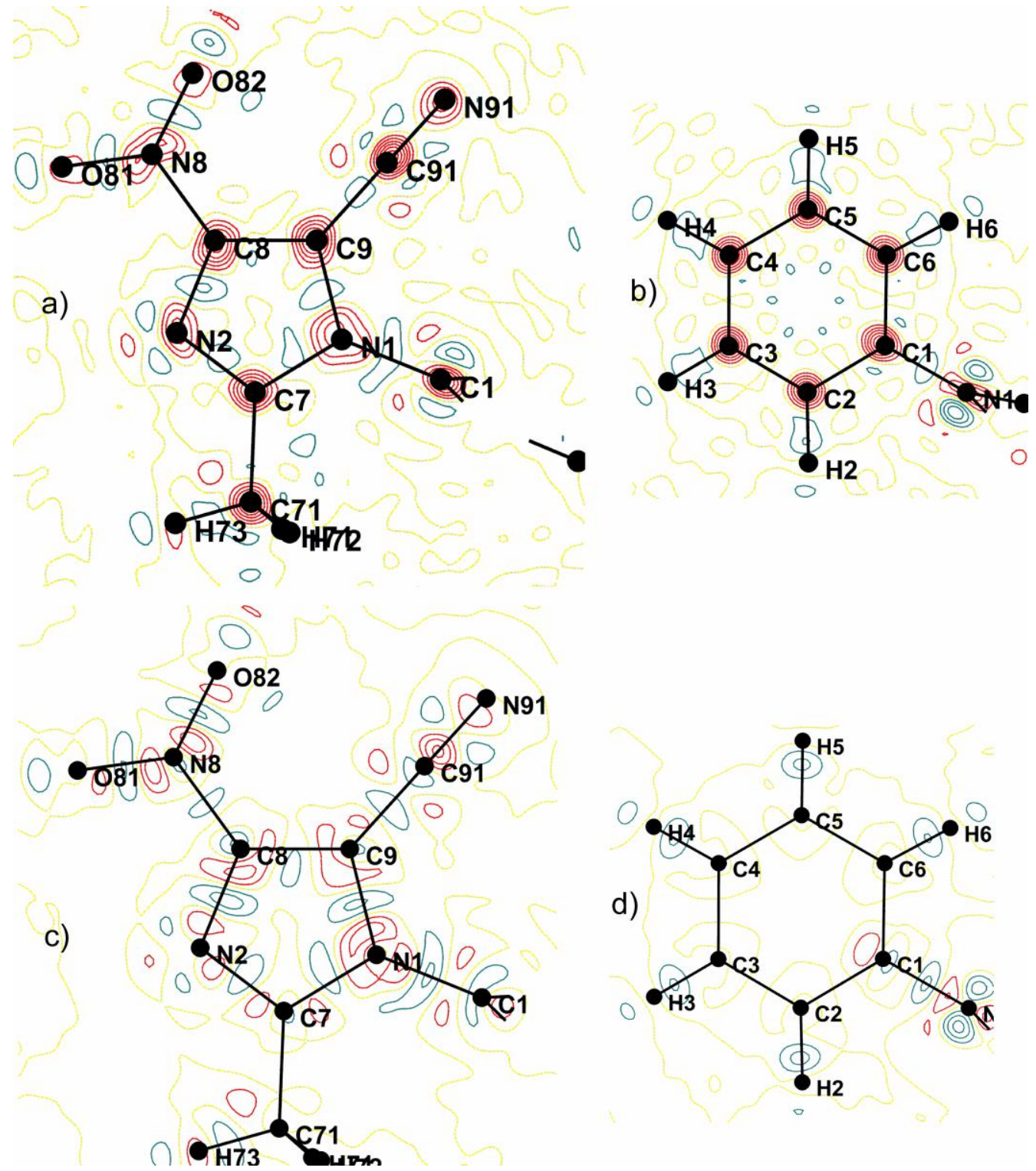

Figure S1. Example of residual Fourier density map for model 1c ( $c f$. Table 1). a), b) without proper core scaling and c), d) after $\kappa_{\text {core }}$ refinement; contours $0.05 \mathrm{e} / \AA^{3}, s_{\max }=1 \AA^{-1}$, red negative, blue positive. 


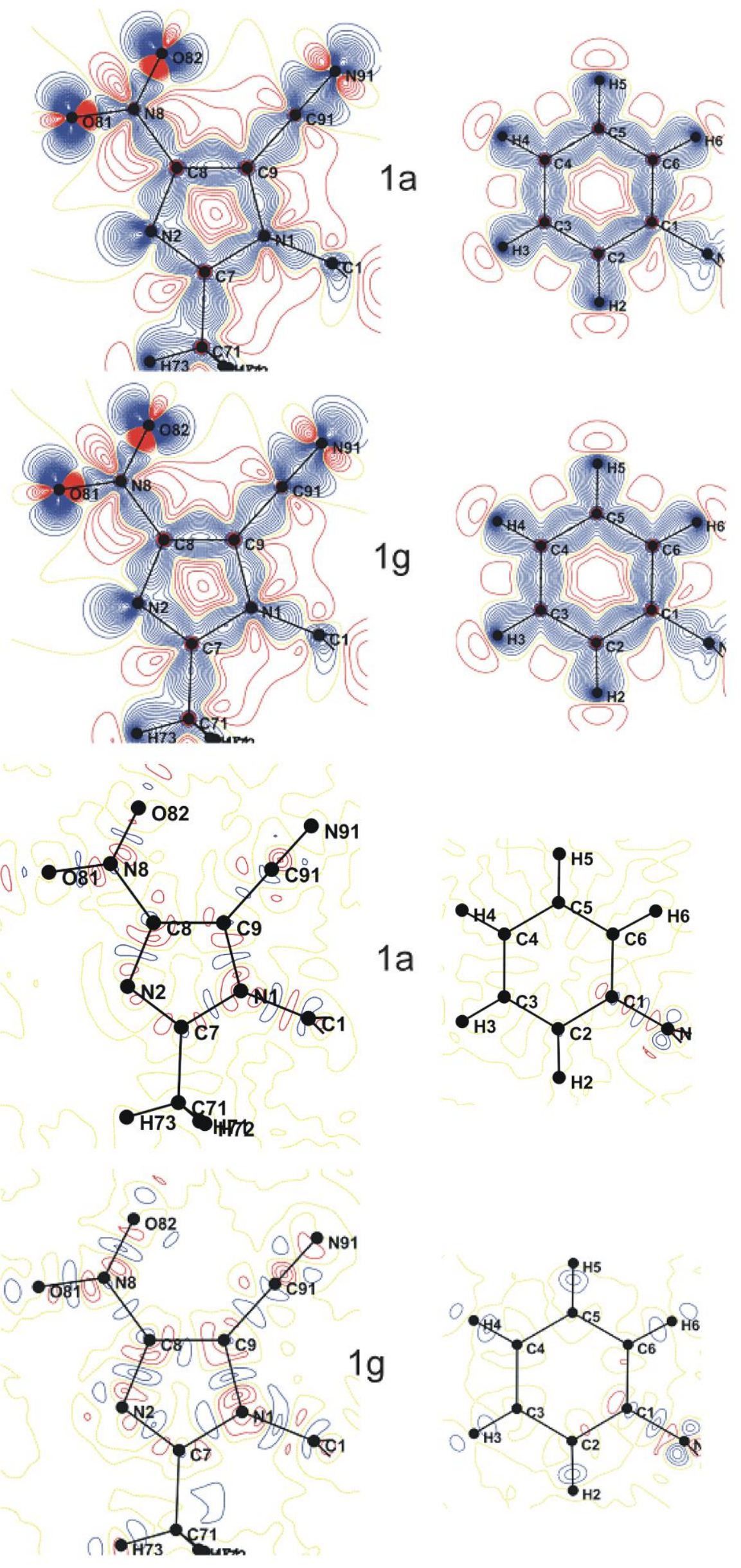


Figure S2. Static deformation and Fourier residual maps for models $1 \mathrm{c}$ and $1 \mathrm{~g}$; contours $\pm 0.05 \mathrm{e} / \mathrm{A}^{3}$, reciprocal resolution up to $s=1 \AA^{-1}$.

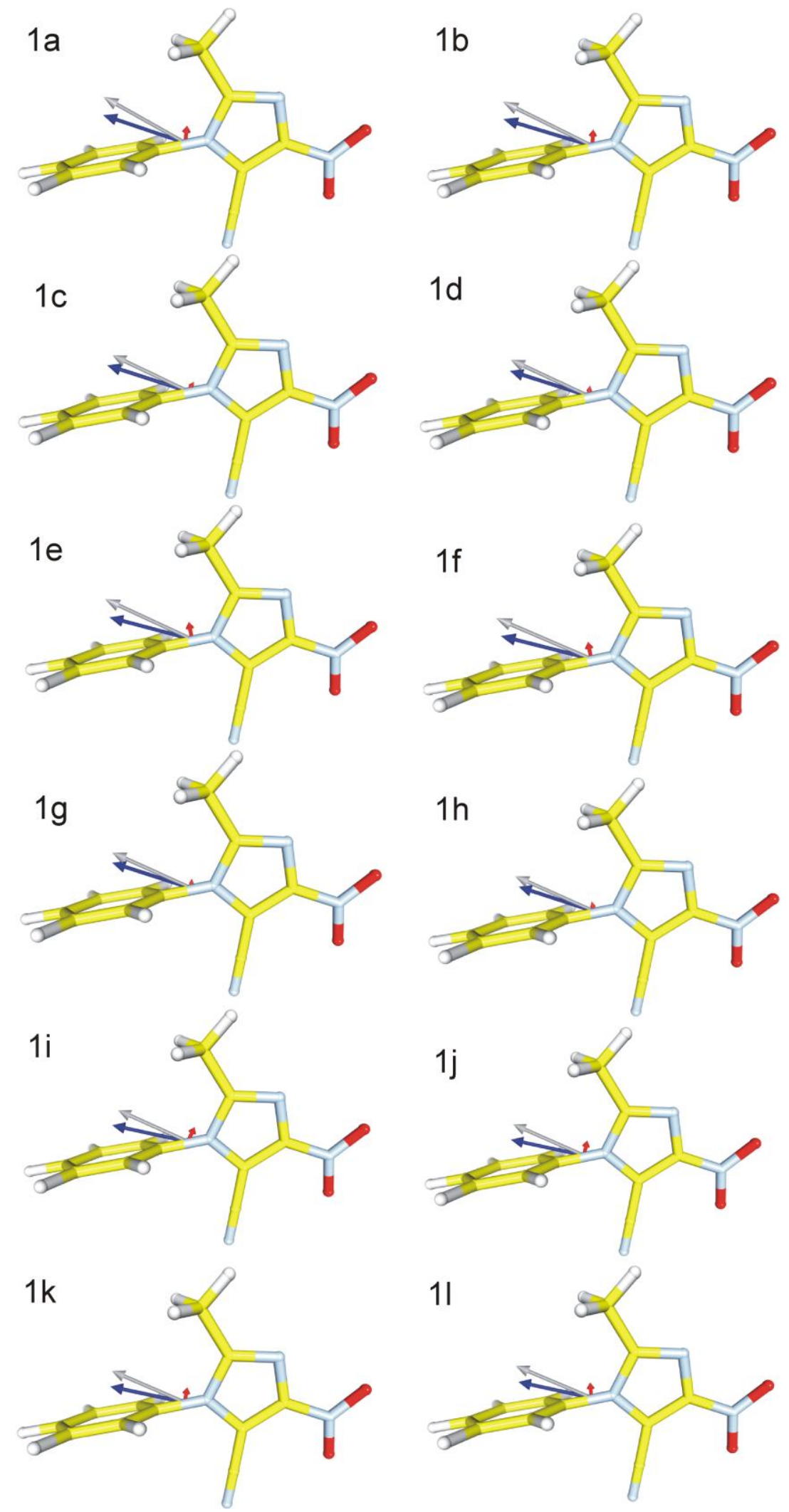

Figure S3. Dipole moment directions (grey - total, red - contribution from atomic dipoles, blue - contribution from atomic charges) for models 1a-11. 


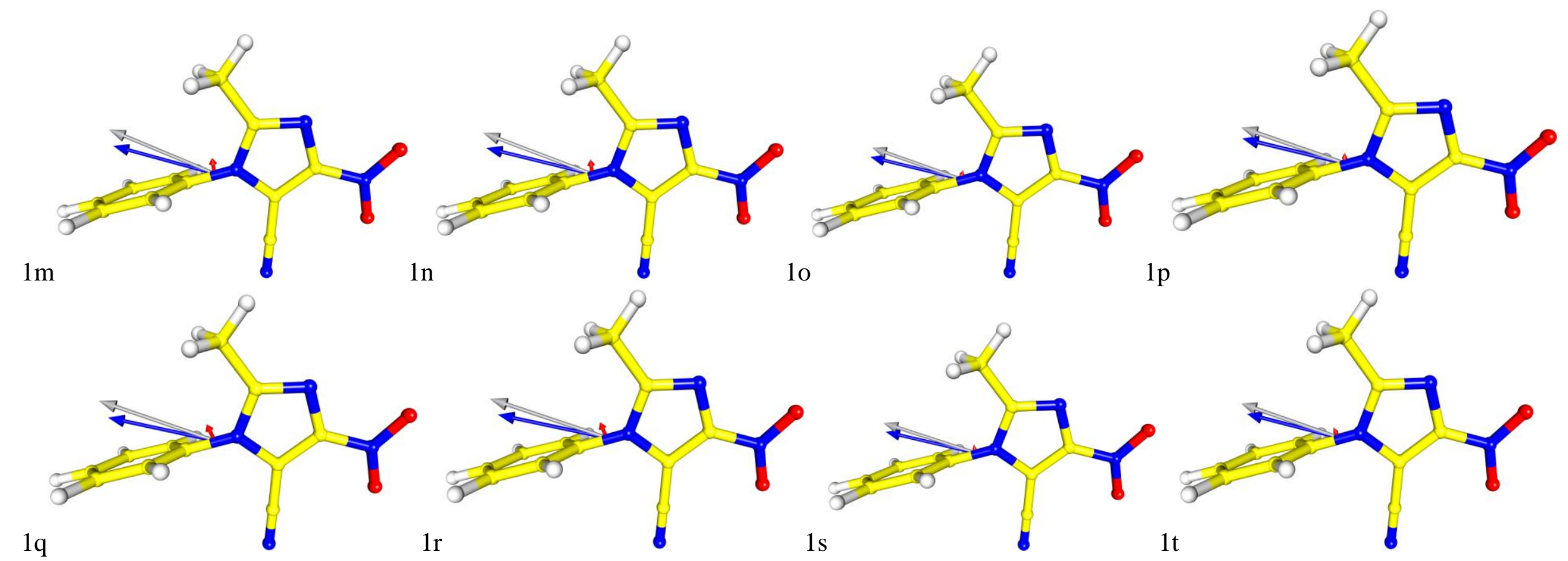

Figure S4. Dipole moments directions (grey - total, red - contribution from atomic dipoles, blue - contribution from atomic charges) for MM with scale factor refined. 


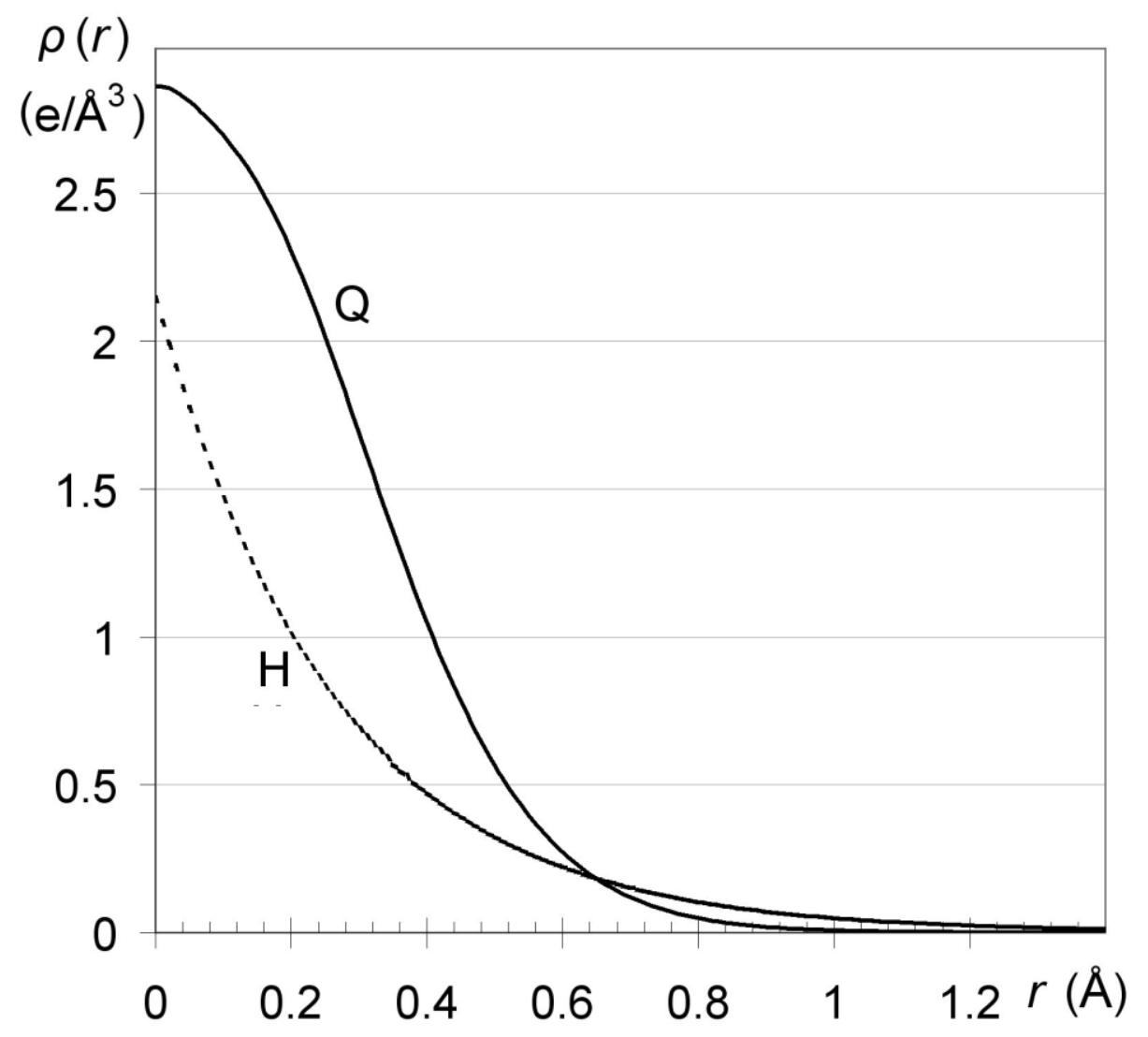

Figure S5. Electron density of a spherical virtual atom with $P_{\mathrm{vir}}=1$ as a function of the distance $r$ to the nucleus. For comparison, the same curve is shown for a hydrogen atom. 
Table S2. Wave function coefficients and orbital exponents for the virtual atom described as a combination of Slater functions. Slater $\left(N_{1}, \xi, r\right)=\operatorname{coeff} * r^{N 1} * \exp (-\xi r)$

\begin{tabular}{|l|l|l|}
\hline$N_{1}$ & $\xi$ & Coeff. \\
\hline 0 & 6.26445 & 0.07360 \\
\hline 1 & 6.06204 & 0.13954 \\
\hline 2 & 3.73854 & 0.87683 \\
\hline
\end{tabular}

Table S3. $\kappa, \mathrm{P}_{\mathrm{val}}, \mathrm{P}_{10}, \mathrm{P}_{11+}$ and $\mathrm{P}_{11-}$ parameters for models 1a-h.

\begin{tabular}{|l|r|r|r|r|r|r|r|r|}
\hline & kp1 & & & & & & \\
\hline & $1 \mathrm{a}$ & $1 \mathrm{~b}$ & $1 \mathrm{c}$ & $1 \mathrm{~d}$ & $1 \mathrm{e}$ & $1 \mathrm{f}$ & $1 \mathrm{~g}$ & $1 \mathrm{~h}$ \\
\hline $\mathrm{C} 1$ & 0.990 & 0.990 & 0.989 & 0.989 & 0.997 & 0.997 & 0.996 & 0.997 \\
\hline C2 & 0.991 & 0.989 & 0.993 & 0.995 & 0.990 & 0.989 & 0.993 & 0.995 \\
\hline C3 & 0.988 & 0.987 & 0.991 & 0.993 & 0.985 & 0.984 & 0.987 & 0.990 \\
\hline C4 & 0.987 & 0.986 & 0.990 & 0.992 & 0.987 & 0.985 & 0.990 & 0.992 \\
\hline C5 & 0.988 & 0.987 & 0.991 & 0.993 & 0.986 & 0.985 & 0.988 & 0.991 \\
\hline C6 & 0.989 & 0.989 & 0.991 & 0.995 & 0.990 & 0.988 & 0.992 & 0.994 \\
\hline C7 & 0.987 & 0.987 & 0.993 & 0.993 & 0.985 & 0.985 & 0.992 & 0.993 \\
\hline C8 & 1.002 & 1.002 & 1.010 & 1.011 & 1.004 & 1.003 & 1.011 & 1.011 \\
\hline C9 & 0.993 & 0.993 & 1.003 & 1.003 & 0.997 & 0.997 & 1.007 & 1.007 \\
\hline C71 & 0.997 & 0.994 & 1.006 & 1.012 & 0.998 & 0.995 & 1.007 & 1.013 \\
\hline C91 & 0.982 & 0.981 & 0.988 & 0.988 & 0.982 & 0.981 & 0.988 & 0.989 \\
\hline N1 & 0.995 & 0.995 & 0.993 & 0.993 & 0.995 & 0.995 & 0.993 & 0.993 \\
\hline N2 & 0.987 & 0.987 & 0.991 & 0.991 & 0.988 & 0.988 & 0.991 & 0.991 \\
\hline N8 & 0.986 & 0.986 & 0.988 & 0.988 & 0.986 & 0.986 & 0.988 & 0.988 \\
\hline N91 & 0.991 & 0.991 & 0.994 & 0.994 & 0.992 & 0.991 & 0.994 & 0.994 \\
\hline O81 & 0.988 & 0.988 & 0.988 & 0.988 & 0.987 & 0.987 & 0.988 & 0.988 \\
\hline O82 & 0.988 & 0.988 & 0.988 & 0.988 & 0.987 & 0.987 & 0.988 & 0.988 \\
\hline H6 & 1.153 & 1.160 & 1.198 & 1.160 & 1.150 & 1.160 & 1.196 & 1.160 \\
\hline H2 & 1.149 & 1.160 & 1.192 & 1.160 & 1.149 & 1.160 & 1.195 & 1.160 \\
\hline H3 & 1.150 & 1.160 & 1.195 & 1.160 & 1.153 & 1.160 & 1.200 & 1.160 \\
\hline H4 & 1.148 & 1.160 & 1.192 & 1.160 & 1.150 & 1.160 & 1.192 & 1.160 \\
\hline
\end{tabular}




\begin{tabular}{|c|c|c|c|c|c|c|c|c|}
\hline $\mathrm{H} 5$ & 1.149 & 1.160 & 1.200 & 1.160 & 1.151 & 1.160 & 1.199 & 1.160 \\
\hline $\mathrm{H} 73$ & 1.149 & 1.160 & 1.184 & 1.160 & 1.151 & 1.160 & 1.187 & 1.160 \\
\hline $\mathrm{H} 72$ & 1.148 & 1.160 & 1.183 & 1.160 & 1.150 & 1.160 & 1.187 & 1.160 \\
\hline \multirow[t]{3}{*}{$\mathrm{H} 71$} & 1.160 & 1.160 & 1.196 & 1.160 & 1.151 & 1.160 & 1.187 & 1.160 \\
\hline & $\mathbf{P}_{\mathrm{val}}$ & & & & & & & \\
\hline & $1 \mathrm{a}$ & $1 b$ & $1 \mathrm{c}$ & $1 d$ & $1 e$ & $1 \mathrm{f}$ & $1 \mathrm{~g}$ & $1 \mathrm{~h}$ \\
\hline C1 & 4.05 & 4.04 & 4.08 & 4.09 & 3.98 & 3.97 & 3.97 & 3.98 \\
\hline $\mathrm{C} 2$ & 4.01 & 4.03 & 4.05 & 4.00 & 4.03 & 4.05 & 4.08 & 4.02 \\
\hline C3 & 4.04 & 4.04 & 4.11 & 4.06 & 4.05 & 4.05 & 4.16 & 4.11 \\
\hline C4 & 4.04 & 4.05 & 4.12 & 4.07 & 4.02 & 4.03 & 4.09 & 4.04 \\
\hline C5 & 4.02 & 4.03 & 4.12 & 4.07 & 4.05 & 4.05 & 4.16 & 4.11 \\
\hline C6 & 4.01 & 4.03 & 4.06 & 4.00 & 4.03 & 4.05 & 4.08 & 4.02 \\
\hline C7 & 4.21 & 4.21 & 4.16 & 4.16 & 4.26 & 4.26 & 4.17 & 4.17 \\
\hline C8 & 3.96 & 3.96 & 3.89 & 3.89 & 3.96 & 3.96 & 3.91 & 3.91 \\
\hline C9 & 3.96 & 3.97 & 3.98 & 3.97 & 3.93 & 3.93 & 3.92 & 3.92 \\
\hline C71 & 3.88 & 3.94 & 4.05 & 3.91 & 3.88 & 3.94 & 4.03 & 3.90 \\
\hline C91 & 4.52 & 4.52 & 4.35 & 4.35 & 4.53 & 4.53 & 4.36 & 4.35 \\
\hline N1 & 4.95 & 4.95 & 5.04 & 5.05 & 4.94 & 4.95 & 5.07 & 5.07 \\
\hline $\mathrm{N} 2$ & 4.96 & 4.97 & 4.97 & 4.97 & 4.94 & 4.95 & 4.97 & 4.97 \\
\hline N8 & 5.03 & 5.03 & 5.01 & 5.01 & 5.03 & 5.03 & 5.01 & 5.01 \\
\hline N91 & 4.69 & 4.70 & 4.82 & 4.82 & 4.67 & 4.68 & 4.82 & 4.83 \\
\hline O81 & 6.14 & 6.14 & 6.14 & 6.15 & 6.14 & 6.15 & 6.14 & 6.14 \\
\hline O82 & 6.13 & 6.14 & 6.14 & 6.14 & 6.14 & 6.15 & 6.14 & 6.14 \\
\hline $\mathrm{H} 6$ & 0.92 & 0.90 & 0.84 & 0.90 & 0.92 & 0.90 & 0.84 & 0.89 \\
\hline $\mathrm{H} 2$ & 0.92 & 0.90 & 0.85 & 0.89 & 0.92 & 0.90 & 0.84 & 0.89 \\
\hline $\mathrm{H} 3$ & 0.93 & 0.91 & 0.85 & 0.90 & 0.91 & 0.90 & 0.83 & 0.88 \\
\hline $\mathrm{H} 4$ & 0.94 & 0.92 & 0.86 & 0.90 & 0.94 & 0.92 & 0.86 & 0.90 \\
\hline $\mathrm{H} 5$ & 0.91 & 0.89 & 0.82 & 0.88 & 0.91 & 0.90 & 0.83 & 0.88 \\
\hline $\mathrm{H} 73$ & 0.95 & 0.93 & 0.91 & 0.95 & 0.94 & 0.92 & 0.90 & 0.95 \\
\hline $\mathrm{H} 72$ & 0.93 & 0.91 & 0.90 & 0.94 & 0.94 & 0.92 & 0.90 & 0.95 \\
\hline $\mathrm{H} 71$ & 0.91 & 0.91 & 0.88 & 0.94 & 0.94 & 0.92 & 0.90 & 0.95 \\
\hline
\end{tabular}

\begin{tabular}{|l|r|r|r|r|r|r|r|r|}
\hline dip x & & & & & & & & \\
\hline C1 & 0.121 & 0.117 & 0.171 & 0.173 & 0.104 & 0.102 & 0.146 & 0.148 \\
\hline
\end{tabular}




\begin{tabular}{|c|c|c|c|c|c|c|c|c|}
\hline $\mathrm{C} 2$ & 0.013 & 0.009 & -0.030 & -0.017 & 0.022 & 0.017 & -0.024 & -0.010 \\
\hline C3 & 0.021 & 0.014 & -0.010 & 0.003 & 0.013 & 0.008 & -0.013 & 0.003 \\
\hline $\mathrm{C} 4$ & 0.022 & 0.016 & -0.010 & 0.003 & 0.020 & 0.013 & -0.015 & -0.002 \\
\hline $\mathrm{C} 5$ & 0.002 & -0.005 & -0.028 & -0.012 & 0.013 & 0.007 & -0.014 & 0.002 \\
\hline C6 & 0.026 & 0.023 & -0.027 & -0.013 & 0.023 & 0.017 & -0.023 & -0.009 \\
\hline $\mathrm{C7}$ & 0.018 & 0.018 & -0.010 & -0.012 & 0.022 & 0.023 & -0.006 & -0.008 \\
\hline $\mathrm{C} 8$ & -0.079 & -0.077 & -0.066 & -0.065 & -0.085 & -0.084 & -0.072 & -0.071 \\
\hline C9 & 0.016 & 0.016 & 0.036 & 0.038 & 0.015 & 0.014 & 0.038 & 0.038 \\
\hline $\mathrm{C} 71$ & 0.018 & 0.013 & 0.017 & 0.013 & 0.001 & 0.001 & 0.002 & 0.002 \\
\hline C91 & -0.003 & -0.004 & 0.012 & 0.013 & 0.000 & 0.000 & 0.001 & 0.001 \\
\hline N1 & 0.003 & 0.003 & 0.014 & 0.016 & -0.002 & -0.002 & 0.015 & 0.015 \\
\hline N2 & -0.138 & -0.138 & -0.126 & -0.126 & -0.140 & -0.140 & -0.125 & -0.125 \\
\hline N8 & -0.027 & -0.027 & -0.021 & -0.021 & -0.026 & -0.027 & -0.020 & -0.021 \\
\hline N91 & -0.001 & -0.001 & 0.000 & 0.000 & 0.000 & 0.000 & 0.000 & 0.000 \\
\hline 081 & -0.116 & -0.116 & -0.115 & -0.115 & -0.116 & -0.116 & -0.115 & -0.115 \\
\hline 082 & -0.116 & -0.116 & -0.116 & -0.115 & -0.116 & -0.115 & -0.115 & -0.115 \\
\hline \multicolumn{9}{|l|}{$\operatorname{dip} y$} \\
\hline $\mathrm{C} 1$ & 0.002 & 0.003 & 0.002 & 0.003 & 0.000 & 0.000 & 0.000 & 0.000 \\
\hline $\mathrm{C} 2$ & 0.030 & 0.028 & 0.032 & 0.032 & 0.001 & 0.001 & 0.001 & 0.001 \\
\hline C3 & 0.009 & 0.009 & 0.009 & 0.007 & 0.000 & 0.000 & 0.000 & 0.000 \\
\hline $\mathrm{C} 4$ & -0.002 & -0.001 & -0.002 & -0.001 & 0.000 & 0.000 & 0.000 & 0.000 \\
\hline C5 & -0.015 & -0.015 & -0.010 & -0.007 & 0.000 & 0.000 & 0.000 & 0.000 \\
\hline C6 & 0.031 & 0.028 & 0.033 & 0.031 & 0.001 & 0.001 & 0.001 & 0.001 \\
\hline $\mathrm{C7}$ & 0.080 & 0.079 & 0.096 & 0.098 & 0.070 & 0.069 & 0.087 & 0.088 \\
\hline $\mathrm{C} 8$ & 0.164 & 0.161 & 0.162 & 0.162 & 0.156 & 0.154 & 0.159 & 0.159 \\
\hline C9 & -0.077 & -0.078 & -0.088 & -0.089 & -0.078 & -0.079 & -0.080 & -0.081 \\
\hline $\mathrm{C} 71$ & -0.006 & -0.006 & -0.004 & -0.004 & 0.000 & 0.000 & 0.000 & 0.000 \\
\hline C91 & 0.008 & 0.008 & 0.007 & 0.008 & 0.001 & 0.001 & 0.001 & 0.001 \\
\hline N1 & -0.017 & -0.017 & -0.014 & -0.013 & -0.001 & -0.001 & -0.001 & -0.001 \\
\hline $\mathrm{N} 2$ & -0.004 & -0.005 & -0.002 & -0.001 & -0.001 & -0.001 & 0.000 & 0.000 \\
\hline N8 & 0.005 & 0.005 & 0.000 & -0.002 & 0.000 & 0.000 & 0.000 & 0.000 \\
\hline N91 & 0.002 & 0.002 & 0.003 & 0.002 & 0.001 & 0.001 & 0.001 & 0.001 \\
\hline O81 & -0.002 & -0.002 & -0.005 & -0.005 & -0.002 & -0.002 & -0.005 & -0.005 \\
\hline O82 & -0.002 & 0.002 & -0.005 & -0.005 & -0.002 & -0.002 & -0.005 & -0.005 \\
\hline
\end{tabular}




\begin{tabular}{|c|c|c|c|c|c|c|c|c|}
\hline $\operatorname{dip} z$ & & & & & & & & \\
\hline $\mathrm{C} 1$ & 0.000 & 0.000 & 0.003 & 0.003 & & & & \\
\hline $\mathrm{C} 2$ & 0.005 & 0.005 & 0.002 & 0.002 & & & & \\
\hline C3 & -0.005 & -0.005 & -0.002 & -0.003 & & & & \\
\hline C4 & -0.001 & -0.001 & 0.002 & 0.002 & & & & \\
\hline C5 & 0.000 & 0.000 & 0.000 & 0.000 & & & & \\
\hline C6 & -0.001 & -0.001 & 0.001 & 0.000 & & & & \\
\hline $\mathrm{C7}$ & 0.006 & 0.006 & 0.006 & 0.006 & & & & \\
\hline C8 & -0.002 & -0.002 & -0.001 & -0.001 & & & & \\
\hline $\mathrm{C9}$ & 0.003 & 0.003 & 0.000 & -0.001 & & & & \\
\hline C71 & -0.065 & -0.068 & -0.091 & -0.082 & -0.067 & -0.071 & -0.086 & -0.078 \\
\hline C91 & 0.385 & 0.382 & 0.316 & 0.312 & 0.387 & 0.384 & 0.306 & 0.302 \\
\hline N1 & -0.004 & -0.004 & -0.003 & -0.002 & -0.001 & -0.001 & 0.000 & 0.000 \\
\hline N2 & 0.001 & 0.001 & 0.001 & 0.001 & 0.000 & 0.000 & 0.000 & 0.000 \\
\hline N8 & -0.002 & -0.002 & -0.003 & -0.003 & 0.000 & 0.000 & 0.000 & 0.000 \\
\hline N91 & -0.133 & -0.133 & -0.120 & -0.119 & -0.133 & -0.133 & -0.119 & -0.118 \\
\hline O81 & 0.000 & 0.000 & 0.001 & 0.001 & 0.000 & 0.000 & 0.000 & 0.000 \\
\hline O82 & -0.001 & -0.001 & -0.001 & -0.001 & 0.000 & 0.000 & 0.000 & 0.000 \\
\hline $\mathrm{H} 6$ & 0.168 & 0.167 & 0.130 & 0.154 & 0.167 & 0.169 & 0.131 & 0.158 \\
\hline $\mathrm{H} 2$ & 0.173 & 0.175 & 0.134 & 0.162 & 0.168 & 0.170 & 0.132 & 0.159 \\
\hline $\mathrm{H} 3$ & 0.163 & 0.165 & 0.125 & 0.151 & 0.167 & 0.170 & 0.125 & 0.154 \\
\hline $\mathrm{H} 4$ & 0.161 & 0.166 & 0.121 & 0.151 & 0.164 & 0.169 & 0.122 & 0.151 \\
\hline $\mathrm{H} 5$ & 0.166 & 0.171 & 0.127 & 0.157 & 0.168 & 0.171 & 0.126 & 0.155 \\
\hline $\mathrm{H} 73$ & 0.157 & 0.150 & 0.112 & 0.133 & 0.151 & 0.146 & 0.112 & 0.135 \\
\hline $\mathrm{H} 72$ & 0.149 & 0.140 & 0.106 & 0.128 & 0.150 & 0.146 & 0.111 & 0.134 \\
\hline $\mathrm{H} 71$ & 0.156 & 0.155 & 0.117 & 0.142 & 0.151 & 0.147 & 0.112 & 0.136 \\
\hline
\end{tabular}




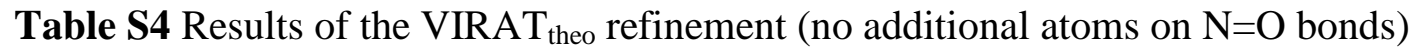

\begin{tabular}{|c|c|c|c|c|c|c|}
\hline Model & Restraints/Constraints & $\begin{array}{l}\text { No } \\
\text { reflections/ } \\
\text { No } \\
\text { parameters }\end{array}$ & $\begin{array}{l}\mathrm{RF} \\
\text { factor } \\
w \mathrm{R} 2 \mathrm{~F} \\
\text { factor }\end{array}$ & Goof & $\begin{array}{l}\text { Dipole } \\
\text { moment } \\
\text { value [D] }\end{array}$ & $\begin{array}{l}\text { Residual } \\
\text { density }\left[\mathrm{e} / \mathrm{A}^{3}\right] \\
\text { up to } 1.2 \AA^{-1}\end{array}$ \\
\hline $2 \mathrm{j}$ & Default restraints* & $\begin{array}{l}15074 \quad / \\
213\end{array}$ & $\begin{array}{l}0.986 \\
1.123\end{array}$ & 0.134 & 17.30 & $\begin{array}{l}0.605(33) / \\
-0.688(33)\end{array}$ \\
\hline $2 \mathrm{k}$ & Modified rest** & $\begin{array}{l}15074 \quad / \\
213\end{array}$ & $\begin{array}{l}0.840 \\
0.987\end{array}$ & 0.118 & 17.33 & $\begin{array}{l}0.329(29) / \\
-0.644(29)\end{array}$ \\
\hline 21 & No restraints & $\begin{array}{l}15074 \\
213\end{array}$ & $\begin{array}{l}0.819 \\
0.977\end{array}$ & 0.117 & 18.70 & $\begin{array}{l}0.297(29) / \\
-0.578(29)\end{array}$ \\
\hline $2 \mathrm{~m}$ & $\begin{array}{l}\text { Modified rest } \kappa_{\mathrm{HYD}}= \\
\mathbf{1 . 1}\end{array}$ & $\begin{array}{l}15074 \\
205\end{array}$ & $\begin{array}{l}0.863 \\
1.018\end{array}$ & 0.122 & 11.30 & $\begin{array}{l}0.322(30) / \\
-0.646(30)\end{array}$ \\
\hline $2 n$ & $\begin{array}{l}\text { Modified rest } \kappa_{\mathrm{HYD}}= \\
\mathbf{1 . 1 3}\end{array}$ & $\begin{array}{l}15074 \\
205\end{array}$ & $\begin{array}{l}0.853 \\
1.004\end{array}$ & 0.120 & 12.84 & $\begin{array}{l}0.324(30) / \\
-0.644(30)\end{array}$ \\
\hline 20 & $\begin{array}{l}\text { Modified rest } \\
\kappa_{\mathrm{HYD}}=\mathbf{1 . 1 6}\end{array}$ & $\begin{array}{l}15074 \quad / \\
205\end{array}$ & $\begin{array}{l}0.847 \\
0.995\end{array}$ & 0.119 & 14.31 & $\begin{array}{l}0.326(29) / \\
-0.644(29)\end{array}$ \\
\hline
\end{tabular}

*Default restraints: Linearity 0.001; Distances: H-Q 0.37 0.01, LP 0.28 0.01; Distance similarities: LP 0.01; Angles LP 0.1; Planarity (LP) 0.001.

**Modified restraints: Linearity 0.1; Distances: H-Q 0.37 0.01, LP 0.28 0.01; Distance similarities: LP 0.01; Planarity (LP) 0.001 


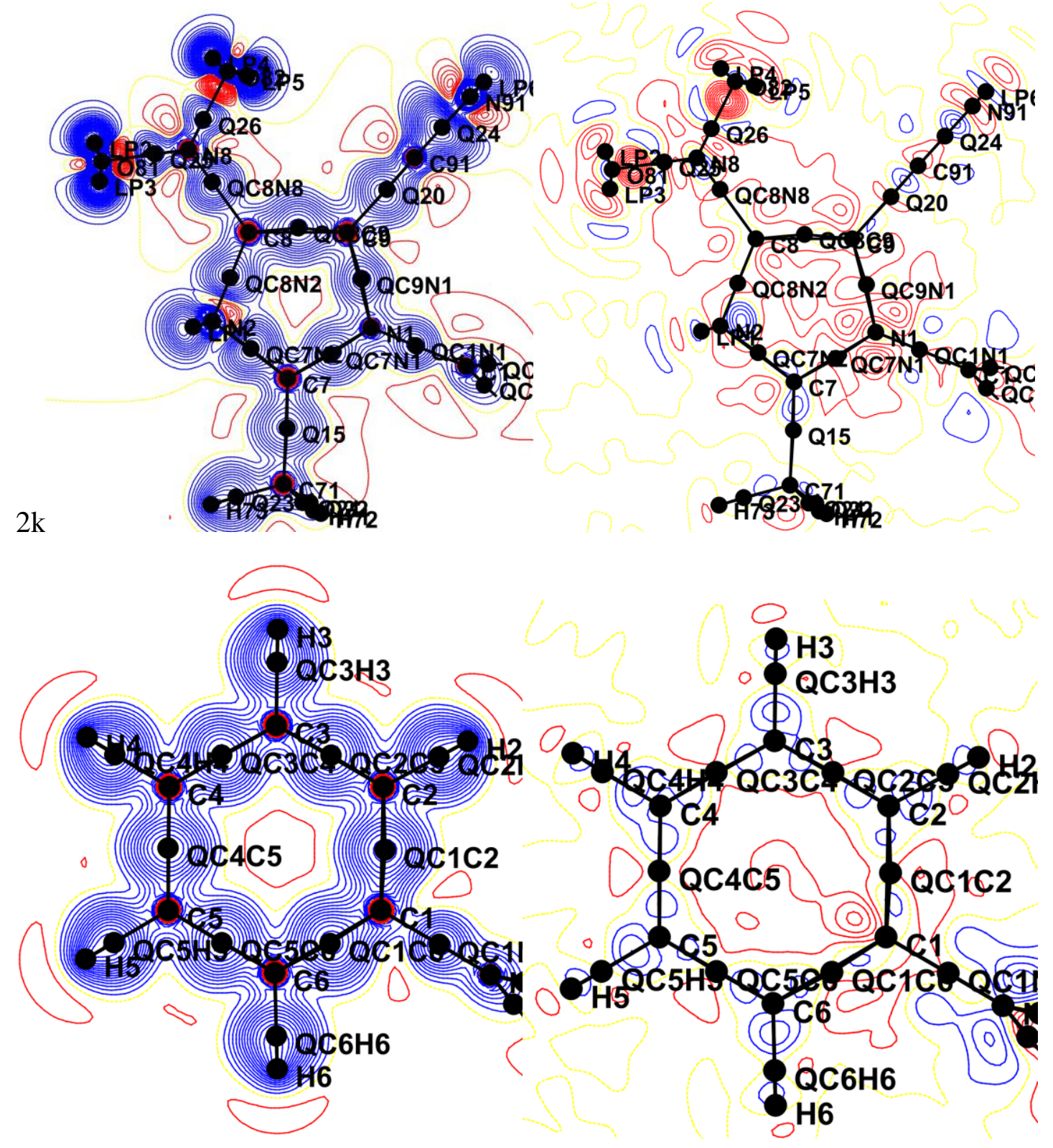




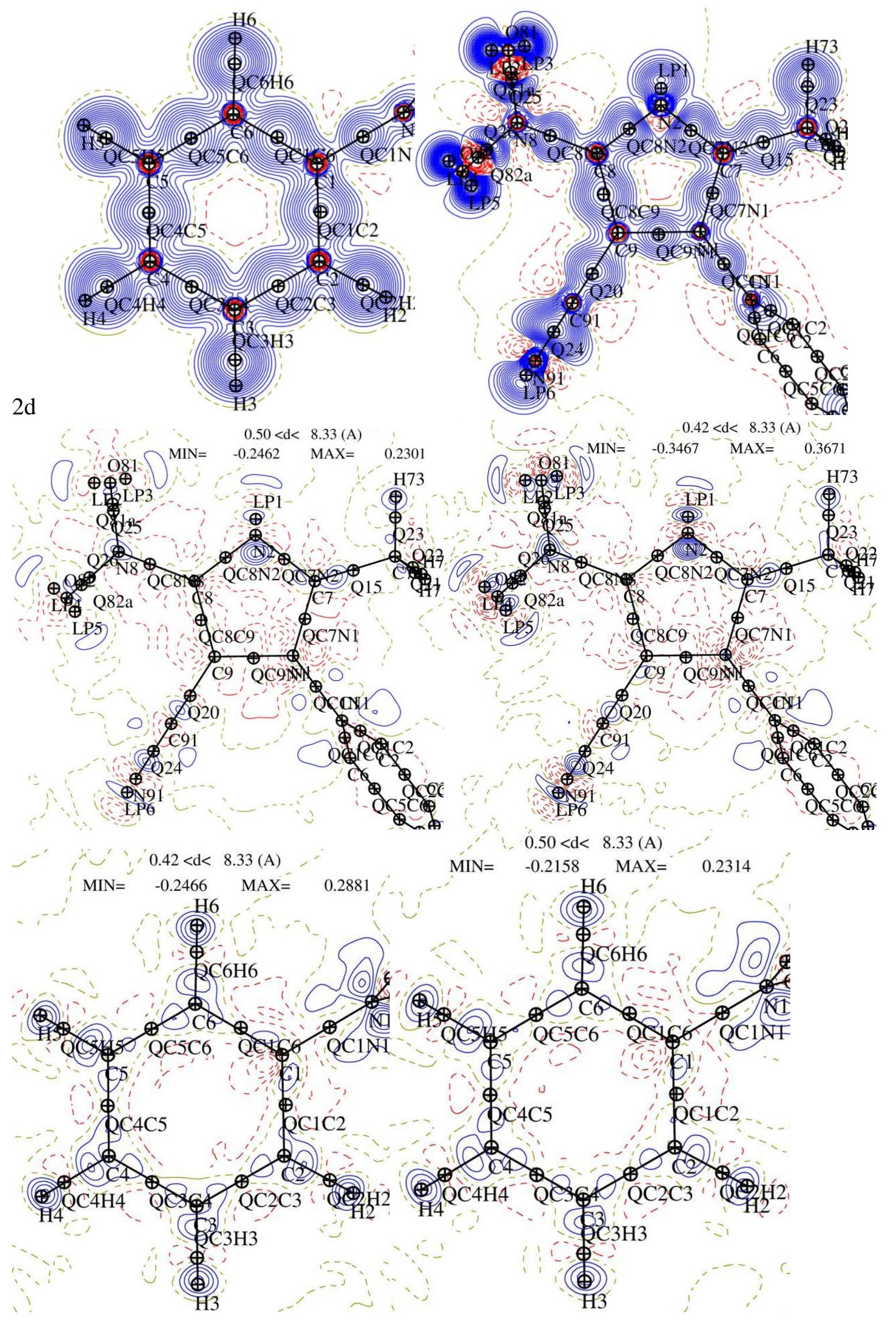



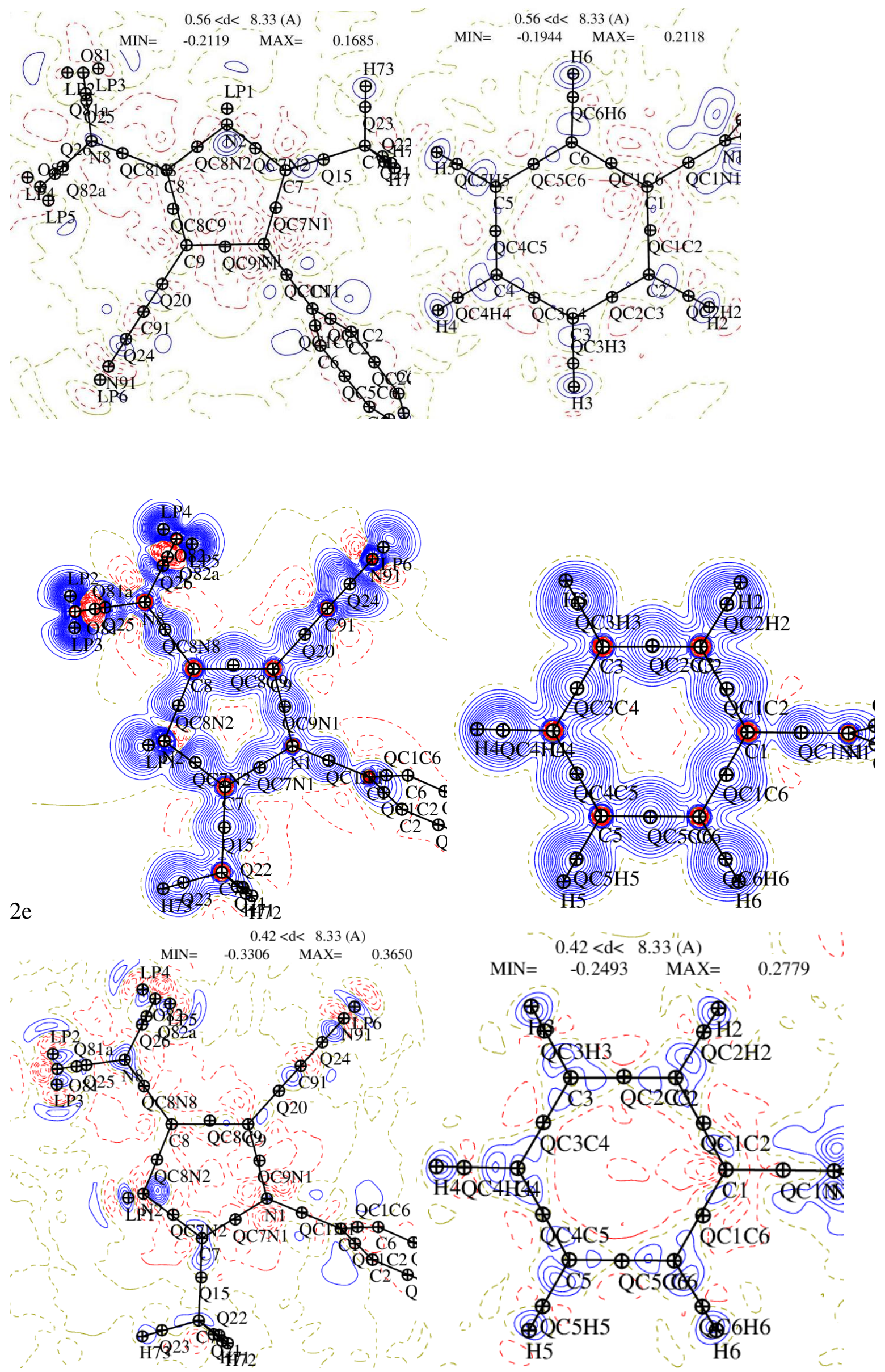

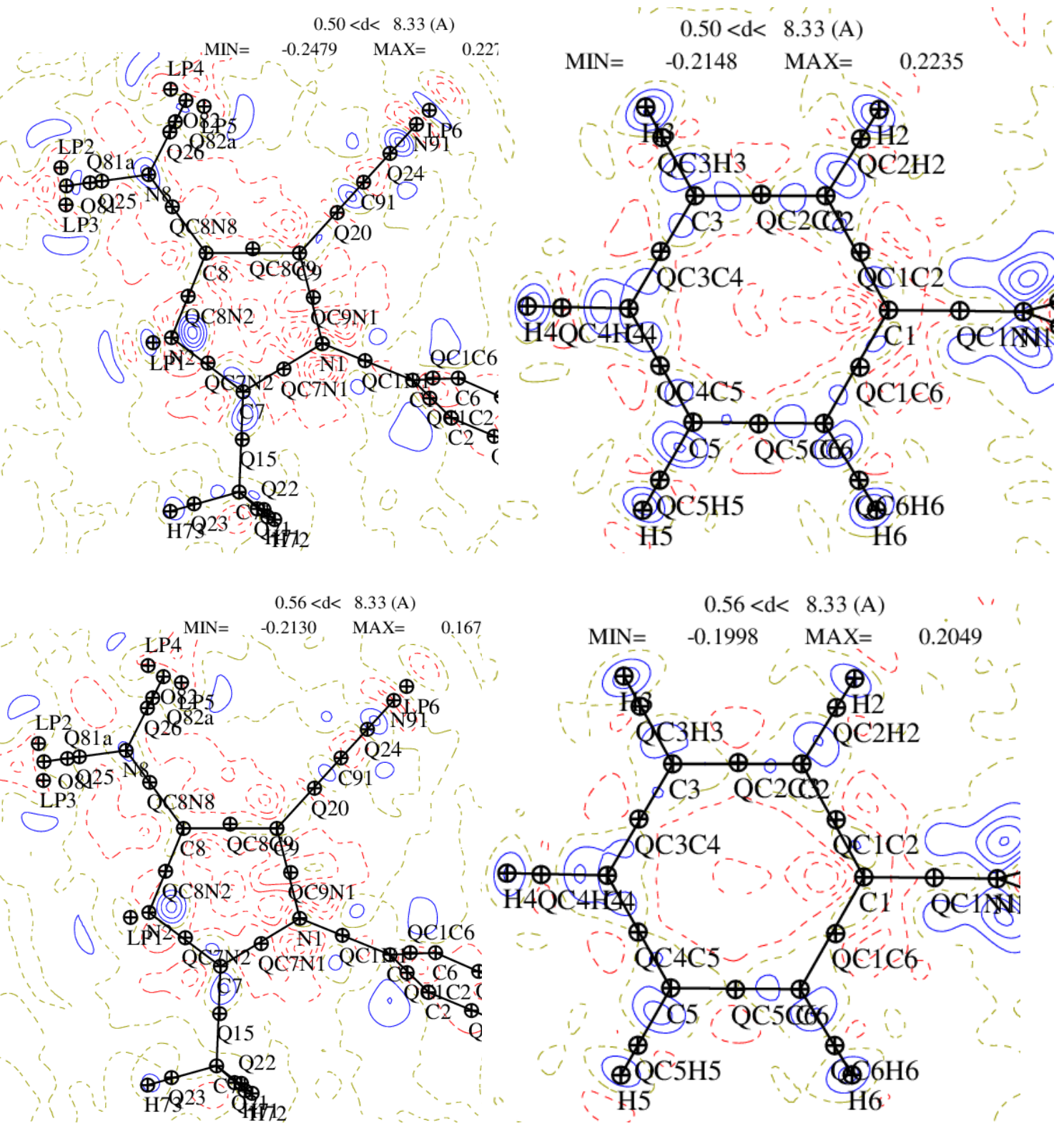

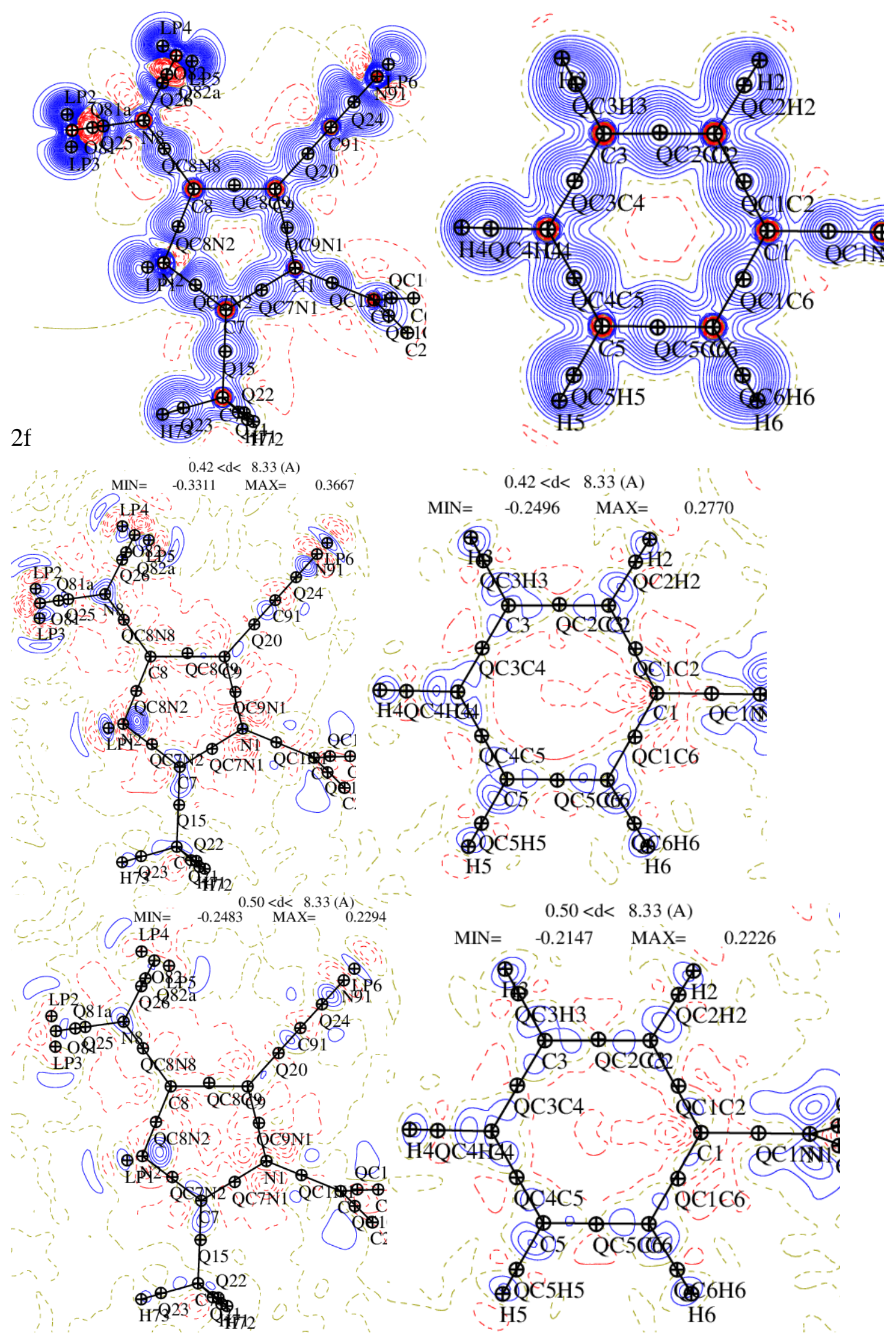


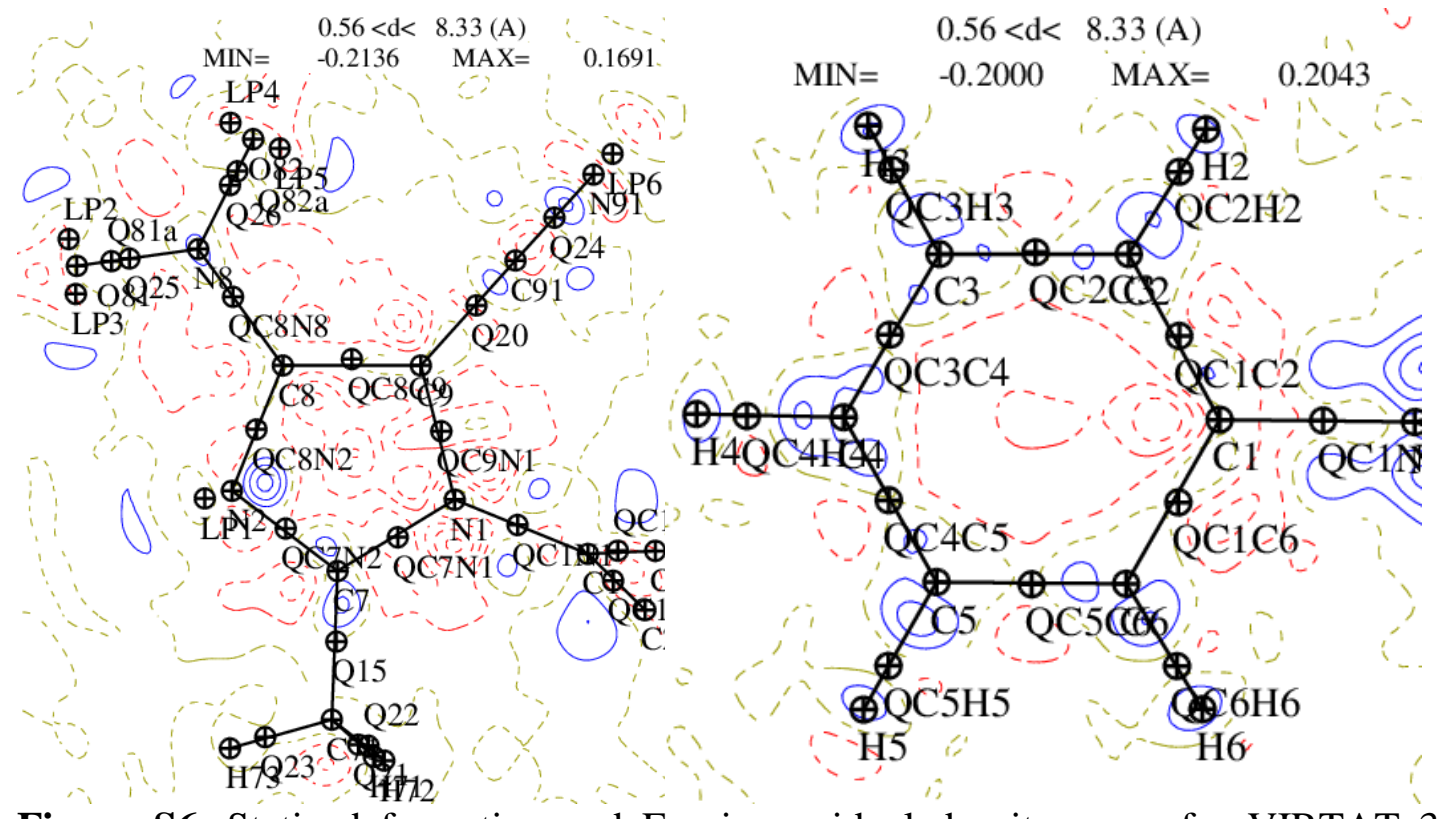

Figure S6. Static deformation and Fourier residual density maps for VIRTAT 3j, 3k, 31, contour $0.05 \mathrm{e} / \mathrm{A}$, resolution $1.2 \mathrm{~A}^{-1}$.

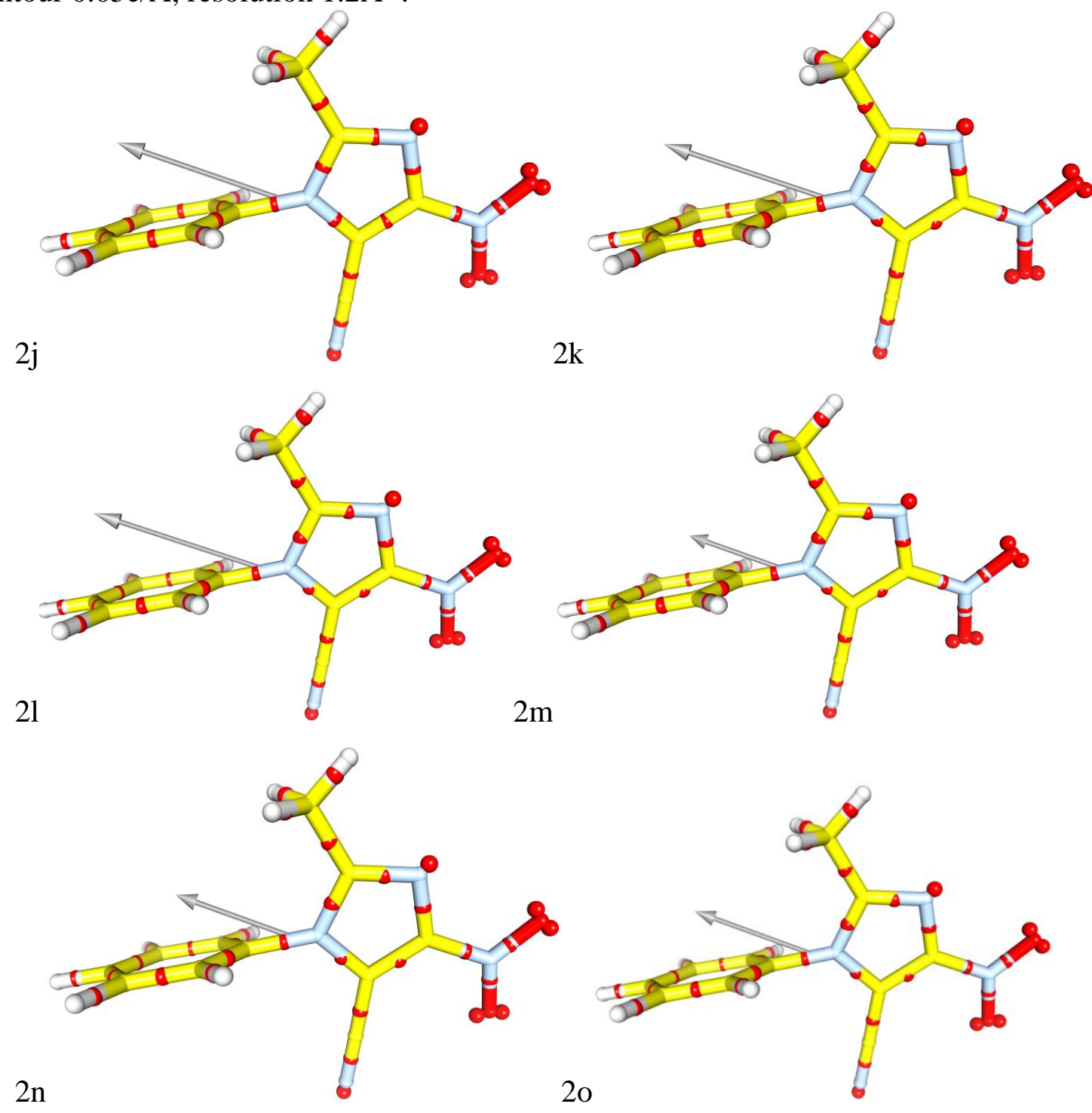

Figure S7. Dipole moment directions in sub-models 2. 
Table S4. $\kappa$ and $\mathrm{P}_{\mathrm{val}}$ for models $2 \mathrm{a}-\mathrm{o}$.

\begin{tabular}{|c|c|c|c|c|c|c|c|c|c|c|c|c|c|c|c|}
\hline & kp1 & & & & & & & & & & & & & & \\
\hline & $2 a$ & $2 b$ & $2 c$ & $2 d$ & $2 e$ & $2 f$ & $2 g$ & $2 \mathrm{~h}$ & $2 \mathrm{i}$ & $2 j$ & $2 k$ & 21 & $2 m$ & $2 n$ & 20 \\
\hline C1 & 1.0387 & 382 & 1.0377 & 1.0387 & 1.0349 & 1.0344 & 1.0386 & \begin{tabular}{|l|}
1.0349 \\
\end{tabular} & 1.0346 & 1.0373 & 1.0379 & 1.0381 & 1.0396 & 1.0390 & 1.0385 \\
\hline $\mathrm{C} 2$ & 312 & 290 & 1.0268 & 311 & 268 & 246 & 1.0302 & 0263 & 1.0240 & 0195 & 1.0236 & 0241 & 1.0331 & 0308 & 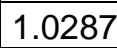 \\
\hline C3 & 94 & 275 & 1.0257 & 2294 & 1.0252 & 0233 & 1.0293 & 0255 & 1.0237 & $\mid 1.0219$ & 1.0229 & 0221 & 0300 & 0281 & 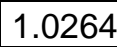 \\
\hline C4 & 557 & 236 & 1.0215 & 257 & 1.0232 & 0212 & 1.0256 & 0232 & 1.0211 & 1.0163 & 1.0171 & .0172 & 1.0256 & 236 & 15 \\
\hline C5 & 93 & 273 & 1.0254 & 0294 & 1.0259 & 1.0240 & 1.0293 & 1.0255 & 1.0237 & 1.0203 & 1.0234 & 1.0212 & 1.0309 & 288 & \\
\hline C6 & 91 & 267 & 1.0246 & 0293 & 1.0258 & 1.0236 & 1.0302 & 263 & 1.0240 & 1.0197 & 1.0214 & 0216 & 303 & 80 & \\
\hline C7 & 28 & 0516 & 1.0506 & 0529 & 1.0509 & 000 & 1.0528 & 510 & 1.0503 & 1.0504 & 1.0487 & 62 & 26 & 13 & \\
\hline C8 & 30 & 20 & 1.0512 & 0530 & 1.0556 & 1.0548 & 1.0532 & 1.0555 & 1.0546 & 1.0566 & 1.0519 & 08 & 56 & 47 & \\
\hline C9 & 34 & 30 & 1.0426 & 0434 & 1.0430 & 26 & 1.0433 & 30 & 28 & \begin{tabular}{|l|l|}
1.0374 \\
\end{tabular} & 1. & 26 & 337 & 34 & \\
\hline C71 & 1. & 20 & 1.0 & 0600 & 1.0 & 66 & 1.0602 & 36 & 65 & \begin{tabular}{|l|}
1.0396 \\
\end{tabular} & 880 & 1.0392 & 612 & 35 & 72 \\
\hline C91 & 72 & 61 & 1.0 & 0070 & 1.0 & 45 & 71 & 56 & 1.0 & 0.9983 & 0.9976 & 973 & 13 & 03 & \\
\hline N1 & 17 & 16 & 1.0116 & 0118 & 1.0108 & 07 & 18 & 08 & 1. & \begin{tabular}{|l|l|}
1.0101 \\
\end{tabular} & 1.0110 & 1.0114 & 14 & 13 & 12 \\
\hline N2 & 549 & 43 & 0.9536 & 0.9549 & 0.9554 & 0.9546 & 0.9551 & 0.9553 & 0.9547 & 0.9527 & 0.9523 & 0.9624 & 549 & 43 & 37 \\
\hline N8 & 36 & 31 & 1.0424 & 0434 & 1.0444 & 1.0437 & 1.0424 & 1.0452 & 1.0445 & 1.0280 & 1.0293 & 1.0337 & 1.0302 & 01 & 96 \\
\hline N91 & 382 & 373 & 0.9364 & 0.9381 & 0.9364 & 0.9355 & 0.9381 & 0.9364 & 0.9354 & 0.9315 & 0.9332 & 0.9388 & 0.9368 & 358 & 350 \\
\hline 081 & 0.9665 & 9653 & 0.9652 & 0.9664 & 0.9660 & 0.9653 & 0.9670 & 0.9660 & 0.9655 & 0.9520 & 0.9408 & 0.9252 & 0.9439 & 0.9428 & 23 \\
\hline 082 & 0.9660 & 9657 & 0.9652 & 0.9663 & 0.9669 & 0.9667 & 0.9670 & 0.9660 & 0.9655 & 0.9518 & 0.9432 & 0.9287 & 0.9458 & 0.9453 & 0.9449 \\
\hline $\mathrm{H} 6$ & 000 & 300 & 1.1600 & 1000 & 1.1300 & 1.1600 & 1.1000 & 1.1300 & 1.1600 & 1.2311 & 1.2335 & 1.2338 & 1.1000 & 1.1300 & 1.1600 \\
\hline $\mathrm{H} 2$ & 000 & 300 & 1.1600 & 1000 & 300 & 1.1600 & 1.1000 & 300 & 1.1600 & 1.2470 & 1.2501 & 1.2559 & 1.1000 & 300 & 1.1600 \\
\hline $\mathrm{H} 3$ & 000 & 300 & 1.1600 & 1000 & 300 & 1600 & 1.1 & 300 & 1.1600 & 1.2361 & 1.2266 & 1.2277 & 1.1000 & 00 & 00 \\
\hline $\mathrm{H} 4$ & 000 & 300 & 1.1600 & 1000 & 300 & 600 & 1.1000 & 300 & 1.1600 & 1.2461 & 1.2433 & 1.2454 & 1.1000 & 300 & 600 \\
\hline $\mathrm{H} 5$ & 000 & 300 & 1.1600 & 1000 & 300 & 300 & 1.1000 & 300 & 1.1600 & 1.2394 & 1.2271 & 1.2311 & 1.1000 & 300 & 600 \\
\hline $\mathrm{H} 73$ & 1000 & 300 & 1.1600 & 1000 & 300 & 500 & 1.1000 & 300 & 1.1600 & 1.2186 & 1.2213 & 1.2320 & 1.1000 & 300 & 1600 \\
\hline $\mathrm{H} 72$ & 000 & 300 & 1.1600 & 1000 & 300 & 600 & 1.1000 & 300 & 1.1600 & \begin{tabular}{|l|}
1.2071 \\
\end{tabular} & 1.1930 & 1.2013 & 1.1000 & 300 & 1.1600 \\
\hline $\mathrm{H} 71$ & 1.1000 & 300 & 1.1600 & 1000 & 300 & 1600 & 1.1000 & 300 & 1.1600 & \begin{tabular}{|l|}
1.2042 \\
\end{tabular} & 1.1953 & 1.1960 & 1.1000 & 1300 & 1.1600 \\
\hline QC1N1 & 1877 & 900 & 1919 & 1877 & 1.2061 & 1.2088 & 1.1877 & 2061 & 1.2084 & 1.2531 & 1.2002 & 1.1971 & 1.1903 & 1.1925 & 1.1953 \\
\hline QC1C6 & 3202 & 239 & 0.8269 & 0.8276 & 8353 & 8385 & 0.8280 & 357 & 0.8389 & 0.8537 & 0.8304 & .8281 & 0.8189 & 225 & 0.8259 \\
\hline QC1C2 & 8452 & 4487 & 0.8523 & 0.8368 & 4402 & 8435 & 365 & 8401 & 0.8434 & 0.8629 & 0.8533 & 0.8527 & 0.8410 & 8450 & 0.8482 \\
\hline QC2C3 & 3347 & 3404 & 0.8454 & 0.8248 & 8389 & 0.8439 & 247 & 92 & 0.8441 & 0.8657 & 0.8564 & 0.8543 & 371 & 431 & 0.8488 \\
\hline QC2H2 & 0.9182 & 0.9123 & 0.9053 & 0.9153 & 0.9169 & 0.9093 & 0.9151 & 0.9169 & 0.9094 & 0.8713 & 0.8760 & 0.8750 & 0.9152 & 9098 & 0.9031 \\
\hline
\end{tabular}


\begin{tabular}{|l|l|l|l|l|l|l|l|l|l|l|l|l|l|l|l|}
\hline QC3C4 & 0.8225 & 0.8280 & 0.8324 & 0.8207 & 0.8338 & 0.8385 & 0.8207 & 0.8341 & 0.8388 & 0.8471 & 0.8380 & 0.8354 & 0.8207 & 0.8258 & 0.8313 \\
\hline QC3H3 & 0.8208 & 0.9147 & 0.9070 & 0.9133 & 0.9049 & 0.8975 & 0.9133 & 0.951 & 0.8976 & 0.8764 & 0.8883 & 0.8886 & 0.9233 & 0.9175 & 0.9095 \\
\hline
\end{tabular} \begin{tabular}{|l|l|l|l|l|l|l|l|l|l|l|l|l|l|l|l|l|l|}
\hline QC3H3 & 0.9208 & 0.9147 & 0.9070 & 0.9133 & 0.9049 & 0.8975 & 0.9133 & 0.9051 & 0.8976 & 0.8764 & 0.8883 & 0.8886 & 0.9233 & 0.9175 & 0.9095 \\
\hline QC4C5 & 0.8141 & 0.894 & 0.8241 & 0.8159 & 0.8323 & 0.8365 & 0.8161 & 0.832 & 0.8365 & 0.8509 & 0.8287 & 0.8246 & 0.8118 & 0.812 & 0.8218 \\
\hline
\end{tabular} \begin{tabular}{|l|l|l|l|l|l|l|l|l|l|l|l|l|l|l|l|l|l|}
\hline QC4C5 & 0.8141 & 0.8194 & 0.8241 & 0.8159 & 0.8323 & 0.8365 & 0.8161 & 0.8324 & 0.8365 & 0.8509 & 0.8287 & 0.8246 & 0.8118 & 0.8172 & 0.8218 \\
\hline
\end{tabular} \begin{tabular}{|l|l|l|l|l|l|l|l|l|l|l|l|l|l|l|l|l|l|l|l|l|}
\hline QC4H4 & 0.9134 & 0.9075 & 0.9004 & 0.9134 & 0.9135 & 0.9066 & 0.9133 & 0.9135 & 0.9067 & 0.8717 & 0.8711 & 0.8715 & 0.9083 & 0.9025 & 0.8958 \\
\hline
\end{tabular} \begin{tabular}{|l|l|l|l|l|l|l|l|l|l|l|l|l|l|l|l|l|l|l|}
\hline QC5C6 & 0.8134 & 0.8189 & 0.8236 & 0.8228 & 0.8320 & 0.8367 & 0.8232 & 0.8324 & 0.8370 & 0.8566 & 0.8347 & 0.8302 & 0.8169 & 0.8224 & 0.8277 \\
\hline
\end{tabular} \begin{tabular}{|l|l|l|l|l|l|l|l|l|l|l|l|l|l|l|l|l|}
\hline QC5H5 & 0.9160 & 0.9107 & 0.9041 & 0.9232 & 0.9180 & 0.9111 & 0.9233 & 0.9182 & 0.9112 & 0.8817 & 0.8860 & 0.8869 & 0.9152 & 0.9101 & 0.9039 \\
\hline QC6H6 & 0.8987 & 0.8923 & 0.8845 & 0.9012 & 0.9056 & 0.8982 & 0.9014 & 0.9059 & 0.8985 & 0.688 & 0.8616 & 0.8628 & 0.9002 & 0.8940 & 0.8852 \\
\hline
\end{tabular} \begin{tabular}{|l|l|l|l|l|l|l|l|l|l|l|l|l|l|l|l|l|l|l|l|}
\hline QC6H6 & 0.8987 & 0.8923 & 0.8845 & 0.9012 & 0.9056 & 0.8982 & 0.9014 & 0.9059 & 0.8985 & 0.8681 & 0.8616 & 0.8628 & 0.9002 & 0.8940 & 0.8852 \\
\hline
\end{tabular} \begin{tabular}{|l|l|l|l|l|l|l|l|l|l|l|l|l|l|l|l|l|l|l|l|}
\hline QC7N1 & 0.9882 & 0.9900 & 0.9918 & 0.9880 & 0.9942 & 0.9965 & 0.9877 & 0.9942 & 0.9963 & 1.0141 & 0.9974 & 0.9957 & 0.9883 & 0.9901 & 0.9935 \\
\hline
\end{tabular} \begin{tabular}{|l|l|l|l|l|l|l|l|l|l|l|l|l|l|l|l|l|l|l|}
\hline QC7N2 & 0.9596 & 0.9626 & 0.9655 & 0.9593 & 0.9628 & 0.9665 & 0.9586 & 0.9632 & 0.9659 & 0.9702 & 0.9652 & 0.9693 & 0.9544 & 0.9577 & 0.9599 \\
\hline
\end{tabular} \begin{tabular}{|l|l|l|l|l|l|l|l|l|l|l|l|l|l|l|l|l|l|l|}
\hline Q15 & 0.8078 & 0.8154 & 0.8214 & 0.8077 & 0.8231 & 0.8299 & 0.8076 & 0.8231 & 0.8298 & 0.8226 & 0.8254 & 0.8267 & 0.8055 & 0.8127 & 0.8178 \\
\hline
\end{tabular} \begin{tabular}{|l|l|l|l|l|l|l|l|l|l|l|l|l|l|l|l|l|l|}
\hline QC8N8 & 1.0415 & 1.0425 & 1.0436 & 1.0419 & 1.0276 & 1.0285 & 1.0431 & 1.0266 & 1.0275 & 1.0269 & 1.0254 & 1.0185 & 1.0236 & 1.0243 & 1.0249 \\
\hline
\end{tabular} \begin{tabular}{|l|l|l|l|l|l|l|l|l|l|l|l|l|l|l|l|l|}
\hline QC8N2 & 1.0082 & 1.0115 & 1.0142 & 1.0080 & 1.0144 & 1.0163 & 1.0090 & 1.0138 & 1.0176 & 1.0307 & 1.0175 & 1.0245 & 1.0044 & 1.0075 & 1.0114 \\
\hline
\end{tabular} \begin{tabular}{|l|l|l|l|l|l|l|l|l|l|l|l|l|l|l|l|l|l|l|}
\hline QC8C9 & 0.8671 & 0.8683 & 0.8697 & 0.8671 & 0.8697 & 0.8714 & 0.8670 & 0.8699 & 0.8715 & 0.8754 & 0.8675 & 0.8672 & 0.8625 & 0.8637 & 0.8644 \\
\hline
\end{tabular}

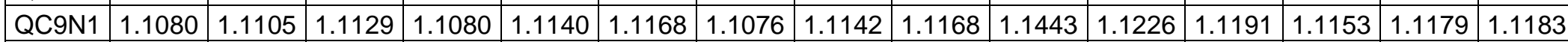
\begin{tabular}{|l|l|l|l|l|l|l|l|l|l|l|l|l|l|l|l|l|l|}
\hline Q20 & 0.8771 & 0.8791 & 0.8808 & 0.8774 & 0.8927 & 0.8943 & 0.8771 & 0.8928 & 0.8943 & 0.8853 & 0.8799 & 0.8808 & 0.8736 & 0.8754 & 0.8770 \\
\hline
\end{tabular} \begin{tabular}{|l|l|l|l|l|l|l|l|l|l|l|l|l|l|l|l|l|l|l|}
\hline Q21 & 0.8687 & 0.8654 & 0.8601 & 0.8747 & 0.8670 & 0.8616 & 0.8745 & 0.8670 & 0.8617 & 0.8331 & 0.8507 & 0.8533 & 0.8689 & 0.8656 & 0.8590 \\
\hline Q22 & 0.8695 & 0.8660 & 0.8596 & 0.8667 & 0.8554 & 0.8496 & 0.8666 & 0.8552 & 0.8495 & 0.899 & 0.8509 & 0.8506 & 0.8691 & 0.8657 & 0.8583 \\
\hline
\end{tabular} \begin{tabular}{|l|l|l|l|l|l|l|l|l|l|l|l|l|l|l|l|l|l|l|}
\hline Q22 & 0.8695 & 0.8660 & 0.8596 & 0.8667 & 0.8554 & 0.8496 & 0.8666 & 0.8552 & 0.8495 & 0.8299 & 0.8509 & 0.8506 & 0.8691 & 0.8657 & 0.8583 \\
\hline
\end{tabular} \begin{tabular}{|l|l|l|l|l|l|l|l|l|l|l|l|l|l|l|l|l|l|l|l|l|l|l|}
\hline Q23 & 0.8676 & 0.8649 & 0.8598 & 0.8642 & 0.8602 & 0.8549 & 0.8641 & 0.8601 & 0.8549 & 0.8426 & 0.8404 & 0.8353 & 0.8674 & 0.8644 & 0.8587 \\
\hline
\end{tabular} \begin{tabular}{|l|l|l|l|l|l|l|l|l|l|l|l|l|l|l|l|l|l|l|}
\hline Q24 & 0.7969 & 0.7994 & 0.8016 & 0.7970 & 0.8000 & 0.8025 & 0.7968 & 0.8001 & 0.8026 & 0.8040 & 0.8031 & 0.8021 & 0.7937 & 0.7962 & 0.7986 \\
\hline
\end{tabular} \begin{tabular}{|l|l|l|l|l|l|l|l|l|l|l|l|l|l|l|l|}
\hline Q25 & 1.2254 & 1.2266 & 1.2309 & 1.2254 & 1.2060 & 1.2091 & 1.2215 & 1.2072 & 1.2096 & 1.2431 & 1.2523 & 1.2306 & 1.2345 & 1.2429 & 1.2469 \\
\hline
\end{tabular} \begin{tabular}{|l|l|l|l|l|l|l|l|l|l|l|l|l|l|l|l|l|l|}
\hline Q26 & 1.2129 & 1.2191 & 1.2230 & 1.2141 & 1.2081 & 1.2123 & 1.2154 & 1.2057 & 1.2104 & 1.2378 & 1.2303 & 1.2121 & 1.2084 & 1.2128 & 1.2193 \\
\hline LP1 & 1.0658 & 1.0688 & 1.071 & 1.0655 & 1.0482 & 1.0501 & 1.0675 & 1.0475 & 1.052 & 1.0614 & 1.0817 & 1.1330 & 1.0695 & 1.0727 & 1.0757 \\
\hline
\end{tabular} \begin{tabular}{|l|l|l|l|l|l|l|l|l|l|l|l|l|l|l|l|}
\hline LP1 & 1.0658 & 1.0688 & 1.0717 & 1.0655 & 1.0482 & 1.0501 & 1.0675 & 1.0475 & 1.0512 & 1.0614 & 1.0817 & 1.1330 & 1.0695 & 1.0727 & 1.0757 \\
\hline
\end{tabular} \begin{tabular}{|l|l|l|l|l|l|l|l|l|l|l|l|l|l|l|l|l|l|l|}
\hline LP2 & 1.4787 & 1.4828 & 1.4866 & 1.4790 & 1.4873 & 1.4935 & 1.4872 & 1.4765 & 1.4808 & 1.5221 & 1.4424 & 1.3996 & 1.4370 & 1.4374 & 1.4388 \\
\hline LP3 & 1.4436 & 1.4472 & 1.4508 & 1.4434 & 1.4533 & 1.4607 & 1.4518 & 1.4429 & 1.4884 & 1.4990 & 1.4239 & 1.3844 & 1.4160 & 1.4182 & 1.4205 \\
\hline
\end{tabular} \begin{tabular}{|l|l|l|l|l|l|l|l|l|l|l|l|l|l|l|l|l|l|l|l}
\hline LP3 & 1.4436 & 1.4472 & 1.4508 & 1.4434 & 1.4533 & 1.4607 & 1.4518 & 1.4429 & 1.4484 & 1.4990 & 1.4239 & 1.3844 & 1.4160 & 1.4182 & 1.4205 \\
\hline
\end{tabular} \begin{tabular}{|l|l|l|l|l|l|l|l|l|l|l|l|l|l|l|l|l|}
\hline LP4 & 1.4973 & 1.5005 & 1.5043 & 1.4975 & 1.4751 & 1.4762 & 1.4874 & 1.4877 & 1.4895 & 1.5117 & 1.4583 & 1.4138 & 1.4500 & 1.4522 & 1.4552 \\
\hline
\end{tabular} \begin{tabular}{|l|l|l|l|l|l|l|l|l|l|l|l|l|l|l|l|l|l|l|l}
\hline LP5 & 1.4696 & 1.4730 & 1.4753 & 1.4702 & 1.4425 & 1.4426 & 1.4584 & 1.4552 & 1.4568 & 1.4727 & 1.4289 & 1.3942 & 1.4240 & 1.4252 & 1.4266 \\
\hline
\end{tabular} \begin{tabular}{|l|l|l|l|l|l|l|l|l|l|l|l|l|l|l|l|l|l|l|l}
\hline LP6 & 1.0295 & 1.0339 & 1.0379 & 1.0300 & 1.0309 & 1.0356 & 1.0297 & 1.0310 & 1.0356 & 1.0362 & 1.0412 & 1.0580 & 1.0256 & 1.0300 & 1.0335 \\
\hline
\end{tabular}

\begin{tabular}{|l|l|l|l|l|l|l|l|l|l|l|}
\hline Q81a & 1.3201 & 1.3570 & 1.3269 & 1.3225 & 1.3709 & 1.3810 & 1.3210 & 1.3500 & 1.3531 \\
\hline Q82a & 1.4370 & 1.4179 & 1.4093 & 1.4223 & 1.3607 & 1.3424 & 1.3431 & 1.4394 & 1.4282 \\
\hline
\end{tabular} \begin{tabular}{|l|l|l|l|l|l|l|l|l|l|}
\hline Q82a & 1.4370 & 1.4179 & 1.4093 & 1.4223 & 1.3607 & 1.3424 & 1.3431 & 1.4394 & 1.4282 \\
\hline
\end{tabular}

\begin{tabular}{|c|c|c|c|c|c|c|c|c|c|c|c|c|c|c|c|}
\hline & val & & & & & & & & & & & & & & \\
\hline & $2 a$ & $2 b$ & $2 c$ & $2 d$ & $2 e$ & $2 f$ & $2 g$ & $2 h$ & $2 \mathrm{i}$ & $2 j$ & $2 \mathrm{k}$ & 21 & $2 m$ & $2 n$ & 20 \\
\hline C1 & 3.3128 & 3.3231 & 3.3328 & 3.3119 & 3.3359 & 3.3456 & 3.3121 & 3.3361 & 3.3457 & 3.4059 & 3.3300 & 3.3251 & 3.2904 & 3.3014 & 3.3118 \\
\hline
\end{tabular}




\begin{tabular}{|c|c|c|c|c|c|c|c|c|c|c|c|c|c|c|c|}
\hline C2 & 2614 & 2956 & 3263 & 3.2085 & 2778 & 3.3076 & 3.2079 & 3.2787 & 3.3088 & 3.3586 & 3.3548 & 3.3515 & 3.2425 & 3.2778 & 30 \\
\hline C3 & 2394 & 2716 & 2971 & 3.2207 & 2763 & 3.3021 & 3.2211 & 2774 & 3.3029 & 3.3480 & 3.3453 & 3.3317 & 3.2479 & 3.2799 & 30 \\
\hline C4 & 08 & 2227 & 2492 & 907 & 2656 & 2921 & 3 & 2661 & 2931 & 3.3130 & 3.2595 & 2467 & 621 & 936 & 22 \\
\hline C5 & 013 & 2350 & 2635 & 3.2207 & 2764 & 3021 & 322 & 2774 & 3.3029 & 3.3589 & 3.3020 & 3.2686 & 1950 & 2288 & 25 \\
\hline C6 & 563 & 1923 & 3.2214 & 3.2083 & 2777 & 3.3075 & 320 & 2787 & 3.3088 & 3.3479 & 3.2619 & 3.2478 & 1580 & 1932 & 22 \\
\hline C7 & 78 & 3.0712 & 3.0833 & 3.0569 & 1086 & 3.1216 & 3.0568 & 1086 & 3.1209 & 3.1406 & 3.0897 & 3.0936 & 413 & 549 & 06 \\
\hline C8 & & 3.3813 & 3.3908 & 3.3703 & 3.3628 & 3.3726 & 3.3690 & & 3.3739 & 3.4067 & 3.3846 & 3.3889 & 425 & 527 & 36 \\
\hline C9 & 98 & 3.4363 & 3.4429 & 3.4301 & 55 & 3.4619 & 3.42 & 59 & 3.4615 & 3.4417 & 3.4388 & 4374 & 59 & 219 & 3.42 \\
\hline C71 & 56 & 2.9224 & 2.9988 & 2.8239 & 05 & 2.9657 & 2.8223 & 0 & 9667 & 2.9534 & 3.0561 & 0706 & 67 & 9006 & 96 \\
\hline C91 & 0149 & 3.0 & 3.0442 & 3.0169 & 53 & 3.0 & 3.0 & 9 & 0795 & 3.0968 & 3.0616 & 76 & 48 & yt & 3.0343 \\
\hline N1 & 9256 & 6 & 9289 & 53 & +5 & 4.9265 & 4.8 & 6 & 9259 & 4.9674 & 4.9441 & 4.9375 & 363 & 4.9377 & 403 \\
\hline N2 & 8015 & 4.8 & +6 & 04 & 1 & 4.7 & $4 . \varepsilon$ & 1 & 8009 & 4.8204 & 4.8646 & 4.9286 & 988 & 4.8162 & 4.8319 \\
\hline N8 & 6 & 4 & 1 & 94 & 3 & 4.0 & 4. & 34 & 0768 & 4.0512 & 4.0400 & 3.9799 & 73 & 4.0237 & 4.0307 \\
\hline N91 & 4 & 4.4721 & .4946 & 4.4498 & 4 & 4.47 & 4.4 & 59 & 4798 & 4.4947 & 4.5173 & 4.5507 & 55 & 4.4500 & 4734 \\
\hline O81 & 630 & 6.0617 & .0802 & 6.0619 & 575 & 6.0787 & 6.0720 & 19 & .0717 & 6.1029 & 5.9665 & 5.9179 & 9297 & 5.9389 & 5.9490 \\
\hline O82 & 586 & 6.0709 & .0812 & 6.0619 & 0675 & 6.0787 & 6.0720 & 19 & .0717 & 6.0920 & 5.9725 & 5.9271 & 5.9424 & 5.9502 & 5.9586 \\
\hline $\mathrm{H} 6$ & 667 & 0.7372 & .7074 & 0.7547 & 0.7327 & 0.7039 & 0.7544 & 7327 & .7038 & 0.6376 & 0.6312 & 0.6391 & .7636 & 0.7345 & 0.7045 \\
\hline $\mathrm{H} 2$ & 428 & 0.7160 & 0.6888 & 0.7544 & 0.7324 & 0.7036 & 0.7544 & 7327 & 7038 & 0.6068 & 0.6023 & 0.5955 & .7388 & .7125 & 0.6859 \\
\hline H3 & 813 & 0.7504 & 0.7195 & 0.7658 & 0.7303 & 0.7002 & 0.7656 & 7303 & .7002 & 0.6369 & 0.6485 & 0.6557 & .7770 & 0.7466 & 0.7162 \\
\hline $\mathrm{H} 4$ & 617 & 0.7315 & 0.7012 & 0.7617 & 0.7366 & 0.7065 & 0.7613 & 7368 & .7066 & 0.6175 & 0.6179 & 0.6227 & .7602 & 0.7303 & 0.7002 \\
\hline $\mathrm{H} 5$ & 501 & 0.7212 & 0.6919 & 0.7656 & 0.7300 & 0.6998 & 0.7656 & 7303 & .7002 & 0.6044 & 0.6247 & 0.6438 & 0.7452 & 0.7165 & 0.6885 \\
\hline $\mathrm{H} 73$ & 3045 & 0.7759 & 0.7453 & 0.7952 & 0.7646 & 0.7346 & 0.7950 & 647 & 7347 & 0.6798 & 0.6792 & 0.6476 & 0.8018 & 0.7729 & .7427 \\
\hline $\mathrm{H} 72$ & 3026 & 0.7739 & 0.7433 & 0.7952 & 0.7649 & 0.7348 & 0.7950 & 7647 & 7347 & 0.6851 & 0.7058 & 0.6894 & 0.7946 & 0.7665 & 0.7362 \\
\hline $\mathrm{H} 71$ & 7792 & 0.7500 & 0.7195 & 0.7951 & 0.7646 & 0.7346 & 0.7950 & 0.7647 & .7347 & 0.6659 & 0.6856 & 0.6978 & 0.7787 & 0.7495 & 0.7187 \\
\hline QC1N1 & 0.1287 & 0.1277 & 0.1268 & 0.1288 & 0.1203 & 0.1194 & 0.1288 & 0.1203 & 1195 & 0.1049 & 0.1241 & 0.1253 & 0.1280 & 1270 & 1259 \\
\hline QC1C6 & 0.5687 & 0.5584 & 0.5501 & 0.5507 & 0.5248 & 0.5164 & 0.5498 & 0.5238 & 5156 & 0.4843 & 0.5440 & 0.5491 & 0.5752 & .5648 & 0.5561 \\
\hline QC1C2 & 0.5106 & 0.5015 & 0.4932 & 0.5289 & 0.5152 & 0.5070 & 0.5298 & 0.5154 & 0.5072 & 0.4725 & 0.4931 & 0.4946 & 0.5236 & 0.5137 & .5055 \\
\hline QC2C3 & 0.5348 & 0.5203 & 0.5082 & 0.5591 & 0.5191 & 0.5073 & 0.5598 & 0.5187 & 0.5070 & 0.4694 & 0.4853 & 0.4897 & 0.5288 & 0.5140 & 0.5012 \\
\hline $\mathrm{QC} 2 \mathrm{H} 2$ & 0.4367 & 0.4388 & 0.4436 & 0.4413 & 0.4269 & 0.4328 & 0.4417 & 0.4268 & 0.4328 & 0.4853 & 0.4842 & 0.4868 & 0.4447 & 0.4461 & 0.4506 \\
\hline QC3C4 & 0.5649 & 0.5501 & 0.5387 & 0.5705 & 0.5349 & 0.5234 & 0.5703 & 0.5342 & 0.5228 & 0.5070 & 0.5274 & 0.5349 & 0.5702 & 0.5558 & 0.5427 \\
\hline QC3H3 & 0.4155 & 0.4183 & 0.4243 & 0.4306 & 0.4381 & 0.4445 & 0.4305 & 0.4378 & 0.4442 & 0.4608 & 0.4453 & 0.4428 & 0.4134 & 0.4157 & 0.4213 \\
\hline QC4C5 & 0.5818 & 0.5665 & 0.5540 & 0.5760 & 0.5273 & 0.5162 & 0.5758 & 0.5272 & 0.5162 & 0.4941 & 0.5461 & 0.5600 & 0.5921 & .5765 & 5634 \\
\hline QC4 & 0.4422 & 0.4452 & 0.4512 & 0.4421 & 0.4342 & 0.4397 & 0.4424 & 0.4341 & 0.4395 & 0.4872 & 0.4925 & 0.4907 & 4514 & 45 & 0.4601 \\
\hline QC5C6 & 0.5817 & 0.5663 & 0.5538 & 0.5571 & 0.5292 & 0.5181 & 0.5561 & 0.5284 & 0.5173 & 0.4830 & 0.5284 & 0.5417 & 0.5725 & 0.5574 & \\
\hline
\end{tabular}




\begin{tabular}{|c|c|c|c|c|c|c|c|c|c|c|c|c|c|c|c|}
\hline $5 \mathrm{H} 5$ & 494 & 4515 & 4568 & 4344 & 4348 & 4410 & .4342 & 0.4346 & 4408 & 0.4822 & 9 & 0.4745 & 4534 & 0.4554 & 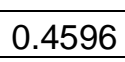 \\
\hline QC6H6 & 4517 & 4554 & 4625 & 4478 & 4279 & 4338 & 4475 & 0.4273 & 4333 & 0.4677 & 14 & 0.4905 & 4513 & 4546 & \\
\hline QC7N1 & 58 & 38 & 521 & 9 & 78 & & 2561 & 478 & 458 & 308 & & 502 & 2558 & 539 & \\
\hline QC7N2 & 0.3389 & 3344 & 301 & 4 & 65 & & 3398 & 662 & 315 & & 4 & 172 & 3457 & 3409 & \\
\hline Q15 & 81 & 5463 & 5295 & 77 & 47 & 81 & 5691 & 48 & 80 & 0.5163 & 11 & & 5766 & 5553 & \\
\hline QC8N8 & 0.2734 & 0.2722 & 0.2708 & 0.27 & 91 & & 0.2717 & 02 & 90 & & & & 034 & 022 & \\
\hline QC8N2 & 0.2 & 0.2 & 0.2 & 0.2 & 13 & & 0.2 & 47 & & & & & & 567 & \\
\hline QC8C9 & 0.4 & 0.4425 & 0.4 & 0.4 & 0.4 & & 0.4 & 34 & & & & & 77 & 547 & \\
\hline QC9N1 & & 0. & & 0.1 & 0. & & 0. & & 0.1 & & & & & 880 & \\
\hline Q20 & & 0.4 & & 0.4 & 0.4 & & 0.4 & 0. & 0.4 & & & & 59 & 721 & \\
\hline Q21 & & 0.4 & 0.4 & $0.4 \varepsilon$ & & & 0.4 & 17 & 0.4909 & & & & 987 & 928 & \\
\hline Q22 & & 0.4 & & 0.46 & 0.4 & & 0.4682 & 99 & 0.4803 & & 34 & 53 & 663 & 594 & \\
\hline Q23 & & 2 & 0.4 & 0.4 & 0. & & 0.4 & 23 & 0.4 & & 0. & 74 & 90 & 319 & \\
\hline Q24 & 24 & & 0.9 & 0.9 & & & 0.9223 & 0.9 & 0.8 & & 97 & 15 & 0.5 & 311 & \\
\hline Q25 & 07 & & 0.4 & 0.4 & & & 0.4 & 0.3 & 0.3 & & 26 & 18 & 0.1 & 366 & \\
\hline Q26 & & 8 & 0.2 & 0.2725 & 0. & & 0. & 0.2657 & 0.2 & & 87 & 0. & 0.1 & 447 & \\
\hline LP1 & & 51 & 0.3 & 0.3289 & 0.3 & & 0.3265 & 0.3 & 0.3 & & 44 & 0. & 0.3 & 0.3226 & \\
\hline LP2 & & & 4 & 0.1 & & & 0.1 & 0.1 & 0.1 & & 0.2 & 0.2 & 0.2236 & 0.2248 & 40 \\
\hline LP3 & & & 0.18 & 0.1 & & & 0.1 & & 0.1 & & 0.2 & 0.2 & 0.2344 & 51 & \\
\hline LP4 & & & 0.16 & 0.1 & & & 0.1 & 0.1 & 0.1 & & & & 0.2 & & \\
\hline LP5 & & & & 0.1 & & & & & 0.1 & & 28 & & 0.2223 & 0.2220 & \\
\hline LP6 & & & & 0. & & & & & 0.4 & & 228 & & 0.4435 & 378 & 327 \\
\hline Q81a & & 3047 & -0.3883 & 3967 & .2603 & -0.2 & 11 & & -0.2 & & & & & & \\
\hline Q82a & -0.1974 & .2156 & -0.2232 & .2097 & -0.2767 & -0.3106 & 3098 & -0.1950 & -0.2 & & & & & & \\
\hline
\end{tabular}




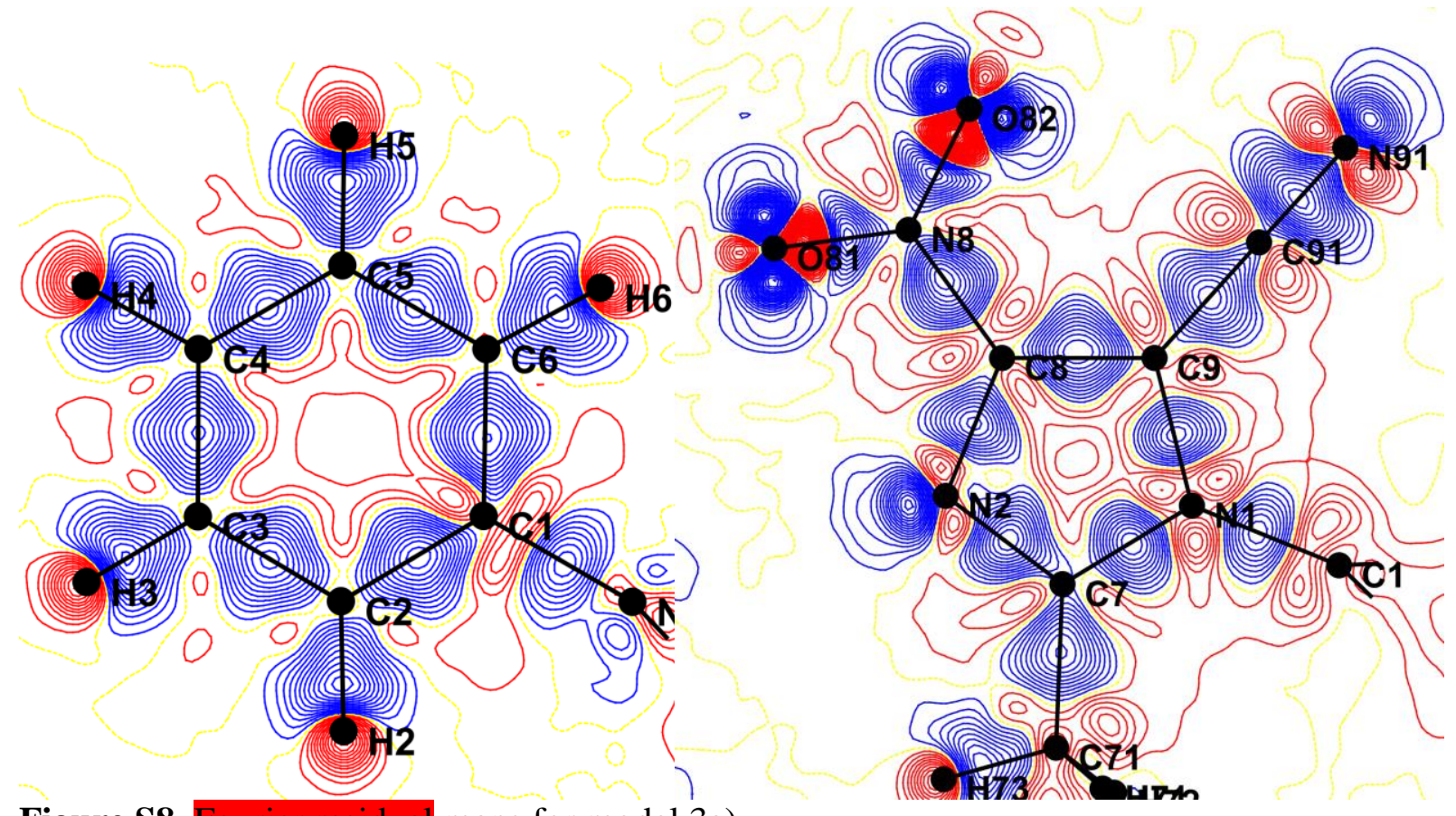

Figure S8. Fourier residual maps for model 3a).

Table S5. $\kappa$ and $\mathrm{P}_{\mathrm{val}}$ for models $3 \mathrm{a}-\mathrm{f}$.

\begin{tabular}{|c|c|c|c|c|c|c|}
\hline & $3 a$ & $3 b$ & $3 c$ & $3 d$ & $3 e$ & $3 f$ \\
\hline & kp1 & & & & & \\
\hline C1 & 1.0376 & 1.0362 & 1.0376 & 1.0382 & 1.0369 & 1.0384 \\
\hline C2 & 1.0218 & 1.0253 & 1.0246 & 1.0227 & 1.0258 & 1.0259 \\
\hline C3 & 1.0217 & 1.0236 & 1.0243 & 1.0225 & 1.0245 & 1.0256 \\
\hline C4 & 1.0201 & 1.0183 & 1.0214 & 1.0212 & 1.0200 & 1.0228 \\
\hline C5 & 1.0217 & 1.0235 & 1.0216 & 1.0225 & 1.0241 & 1.0229 \\
\hline C6 & 1.0218 & 1.0225 & 1.0209 & 1.0227 & 1.0230 & 1.0219 \\
\hline $\mathrm{C7}$ & 1.0371 & 1.0365 & 1.0356 & 1.0385 & 1.0381 & 1.0371 \\
\hline C8 & 1.0375 & 1.0372 & 1.0377 & 1.0393 & 1.0390 & 1.0401 \\
\hline C9 & 1.0384 & 1.0380 & 1.0382 & 1.0392 & 1.0388 & 1.0395 \\
\hline C71 & 1.0101 & 1.0104 & 1.0138 & 1.0113 & 1.0116 & 1.0153 \\
\hline C91 & 1.0411 & 1.0407 & 1.0413 & 1.0421 & 1.0415 & 1.0432 \\
\hline N1 & 1.0164 & 1.0160 & 1.0159 & 1.0170 & 1.0167 & 1.0163 \\
\hline $\mathrm{N} 2$ & 0.9988 & 0.9985 & 0.9995 & 0.9993 & 0.9992 & 1.0005 \\
\hline N8 & 1.0192 & 1.0188 & 1.0166 & 1.0199 & 1.0198 & 1.0174 \\
\hline N91 & 0.9943 & 0.9942 & 0.9951 & 0.9948 & 0.9947 & 0.9963 \\
\hline O81 & 0.9952 & 0.9963 & 0.9961 & 0.9956 & 0.9967 & 0.9965 \\
\hline O82 & 0.9952 & 0.9951 & 0.9957 & 0.9956 & 0.9956 & 0.9960 \\
\hline $\mathrm{H} 6$ & 1.4000 & 1.4000 & 1.4000 & 1.3353 & 1.3300 & 1.3206 \\
\hline $\mathrm{H} 2$ & 1.4000 & 1.4000 & 1.4000 & 1.3353 & 1.3292 & 1.3179 \\
\hline $\mathrm{H} 3$ & 1.4000 & 1.4000 & 1.4000 & 1.3330 & 1.3283 & 1.3221 \\
\hline $\mathrm{H} 4$ & 1.4000 & 1.4000 & 1.4000 & 1.3298 & 1.3302 & 1.3173 \\
\hline $\mathrm{H} 5$ & 1.4000 & 1.4000 & 1.4000 & 1.3330 & 1.3276 & 1.3195 \\
\hline $\mathrm{H} 73$ & 1.4000 & 1.4000 & 1.4000 & 1.3454 & 1.3391 & 1.3336 \\
\hline $\mathrm{H} 72$ & 1.4000 & 1.4000 & 1.4000 & 1.3454 & 1.3384 & 1.3246 \\
\hline $\mathrm{H} 71$ & 1.4000 & 1.4000 & 1.4000 & 1.3454 & 1.3386 & 1.3290 \\
\hline
\end{tabular}

\begin{tabular}{|l|l|l|l|l|l|l|}
\hline & 3a & $3 \mathrm{~b}$ & $3 \mathrm{c}$ & $3 \mathrm{~d}$ & $3 \mathrm{e}$ & $3 \mathrm{f}$ \\
\hline val & & & & & \\
\hline
\end{tabular}




\begin{tabular}{|l|r|r|r|r|r|r|}
\hline C1 & 3.979 & 3.998 & 3.987 & 3.971 & 3.987 & 3.984 \\
\hline C2 & 4.226 & 4.206 & 4.226 & 4.197 & 4.178 & 4.192 \\
\hline C3 & 4.208 & 4.191 & 4.187 & 4.186 & 4.171 & 4.154 \\
\hline C4 & 4.218 & 4.236 & 4.211 & 4.195 & 4.211 & 4.176 \\
\hline C6 & 4.208 & 4.191 & 4.214 & 4.186 & 4.171 & 4.184 \\
\hline C7 & 4.226 & 4.206 & 4.211 & 4.197 & 4.178 & 4.181 \\
\hline C8 & 3.869 & 3.873 & 3.884 & 3.855 & 3.859 & 3.867 \\
\hline C9 & 4.043 & 4.045 & 4.038 & 4.022 & 4.021 & 4.005 \\
\hline C71 & 3.996 & 4.000 & 4.000 & 3.989 & 3.992 & 3.985 \\
\hline C91 & 4.614 & 4.610 & 4.576 & 4.559 & 4.548 & 4.509 \\
\hline N1 & 4.008 & 4.013 & 4.005 & 3.993 & 3.992 & 3.978 \\
\hline N2 & 5.009 & 5.011 & 5.009 & 5.003 & 5.003 & 5.004 \\
\hline N8 & 5.226 & 5.229 & 5.219 & 5.214 & 5.215 & 5.196 \\
\hline N91 & 4.660 & 4.665 & 4.687 & 4.651 & 4.656 & 4.678 \\
\hline O81 & 5.221 & 5.226 & 5.219 & 5.208 & 5.211 & 5.195 \\
\hline O82 & 6.181 & 6.174 & 6.183 & 6.173 & 6.166 & 6.175 \\
\hline H6 & 6.181 & 6.173 & 6.166 & 6.173 & 6.166 & 6.160 \\
\hline H2 & 0.748 & 0.752 & 0.759 & 0.788 & 0.795 & 0.810 \\
\hline H3 & 0.748 & 0.751 & 0.747 & 0.788 & 0.794 & 0.799 \\
\hline H4 & 0.747 & 0.751 & 0.760 & 0.787 & 0.794 & 0.809 \\
\hline H5 & 0.744 & 0.743 & 0.749 & 0.788 & 0.788 & 0.803 \\
\hline H73 & 0.747 & 0.751 & 0.748 & 0.787 & 0.794 & 0.798 \\
\hline H72 & 0.731 & 0.736 & 0.750 & 0.763 & 0.772 & 0.794 \\
\hline H71 & 0.731 & 0.734 & 0.732 & 0.763 & 0.771 & 0.782 \\
\hline & 0.731 & 0.735 & 0.736 & 0.763 & 0.771 & 0.783 \\
\hline
\end{tabular}

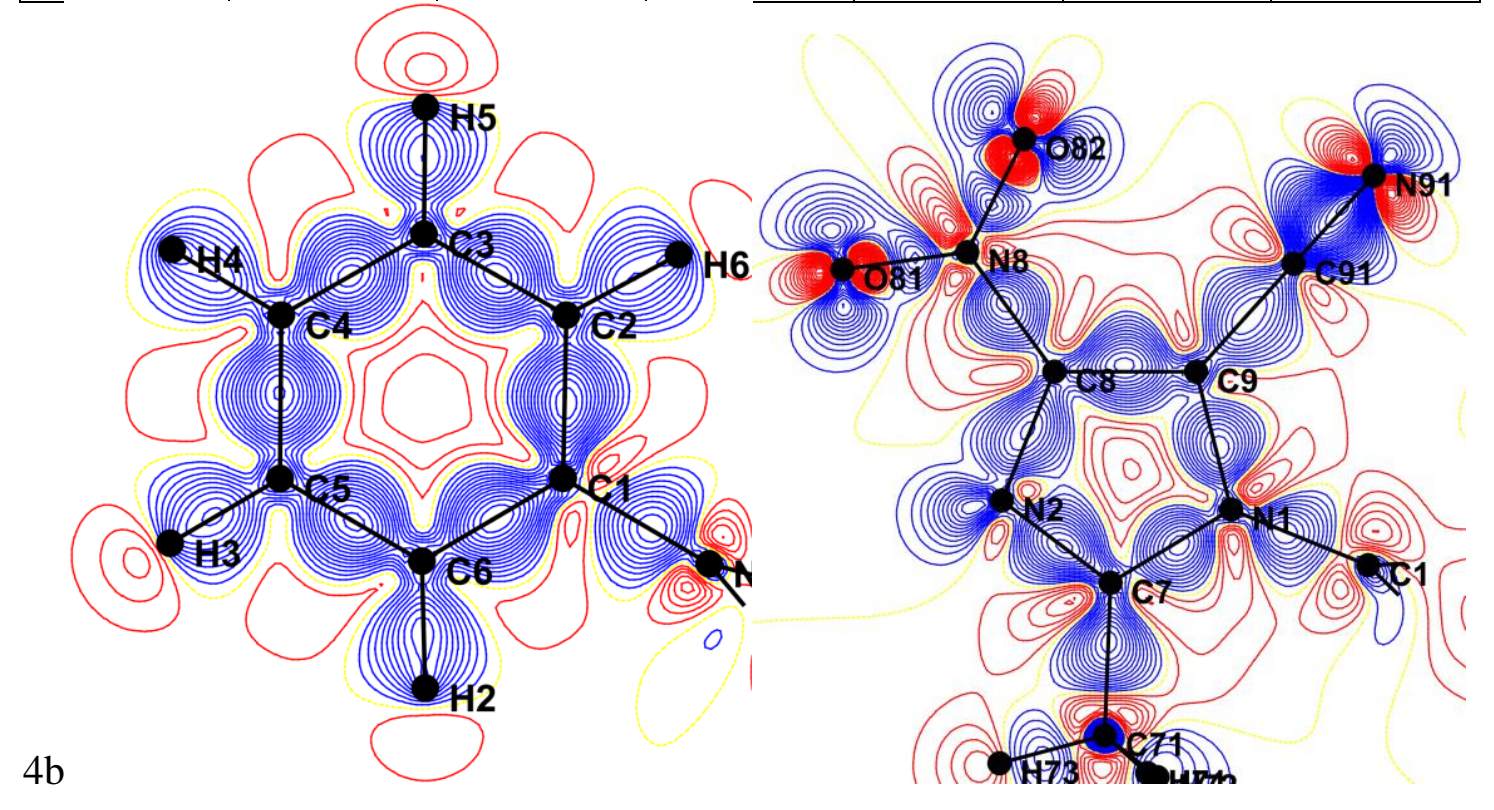




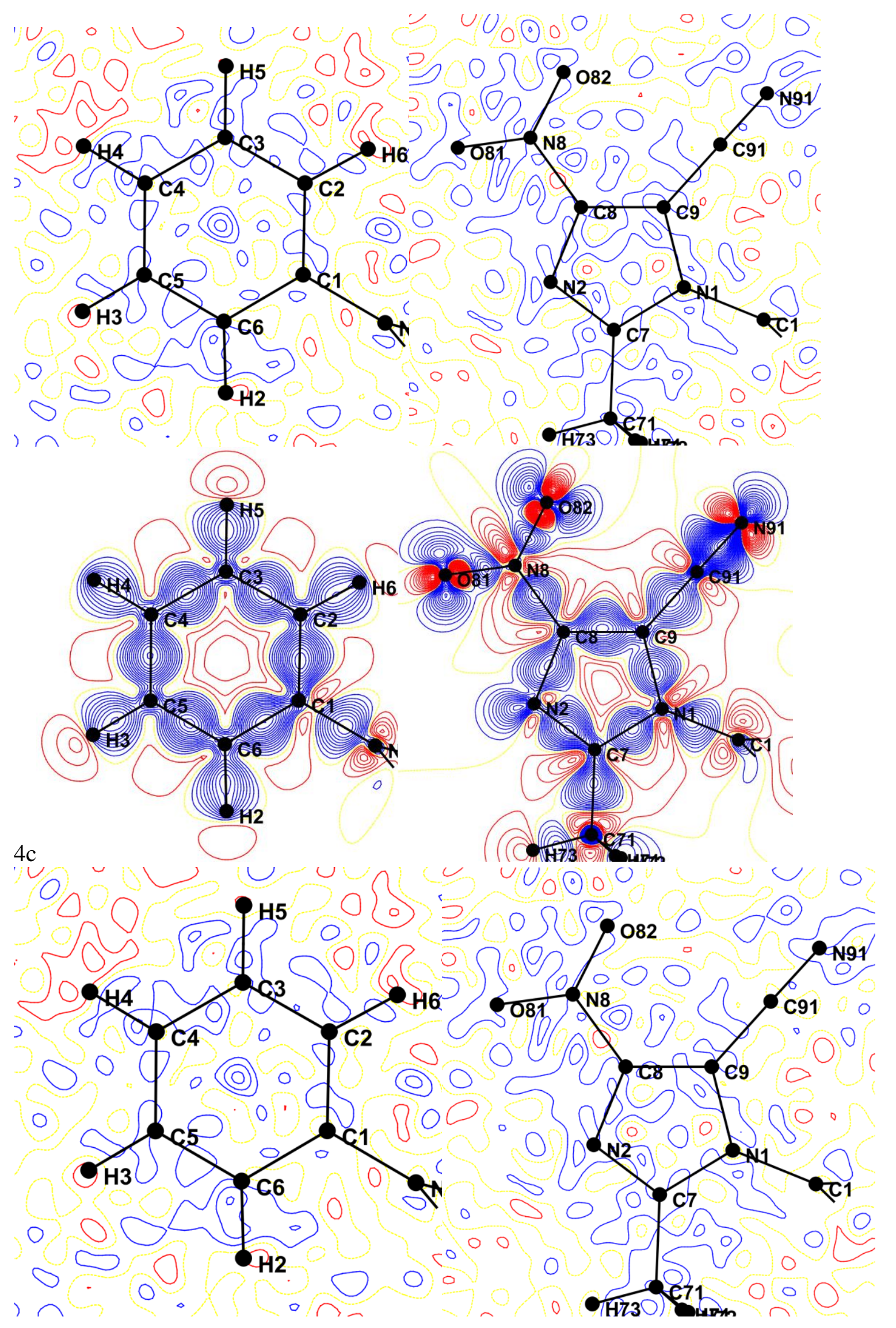




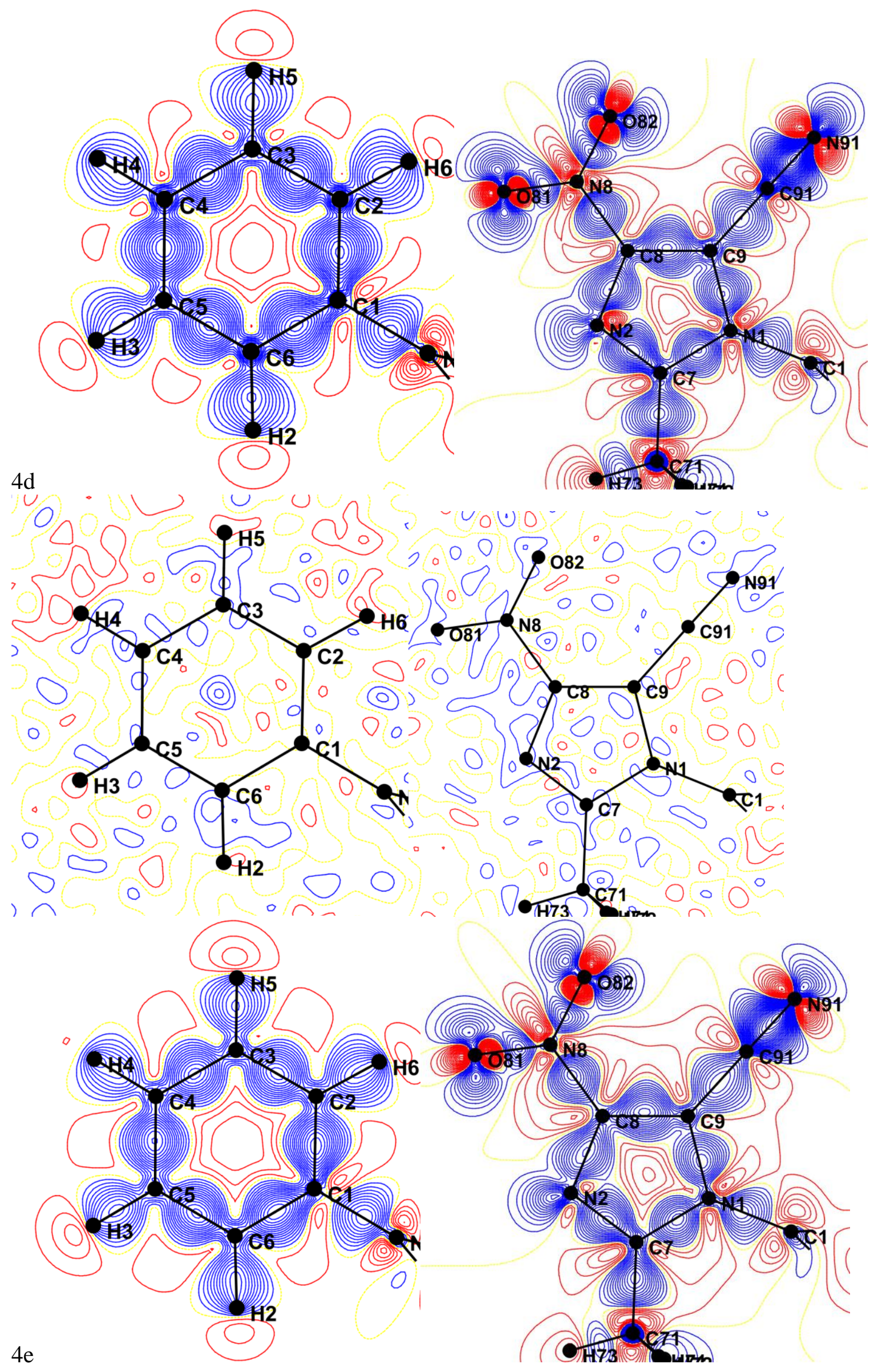




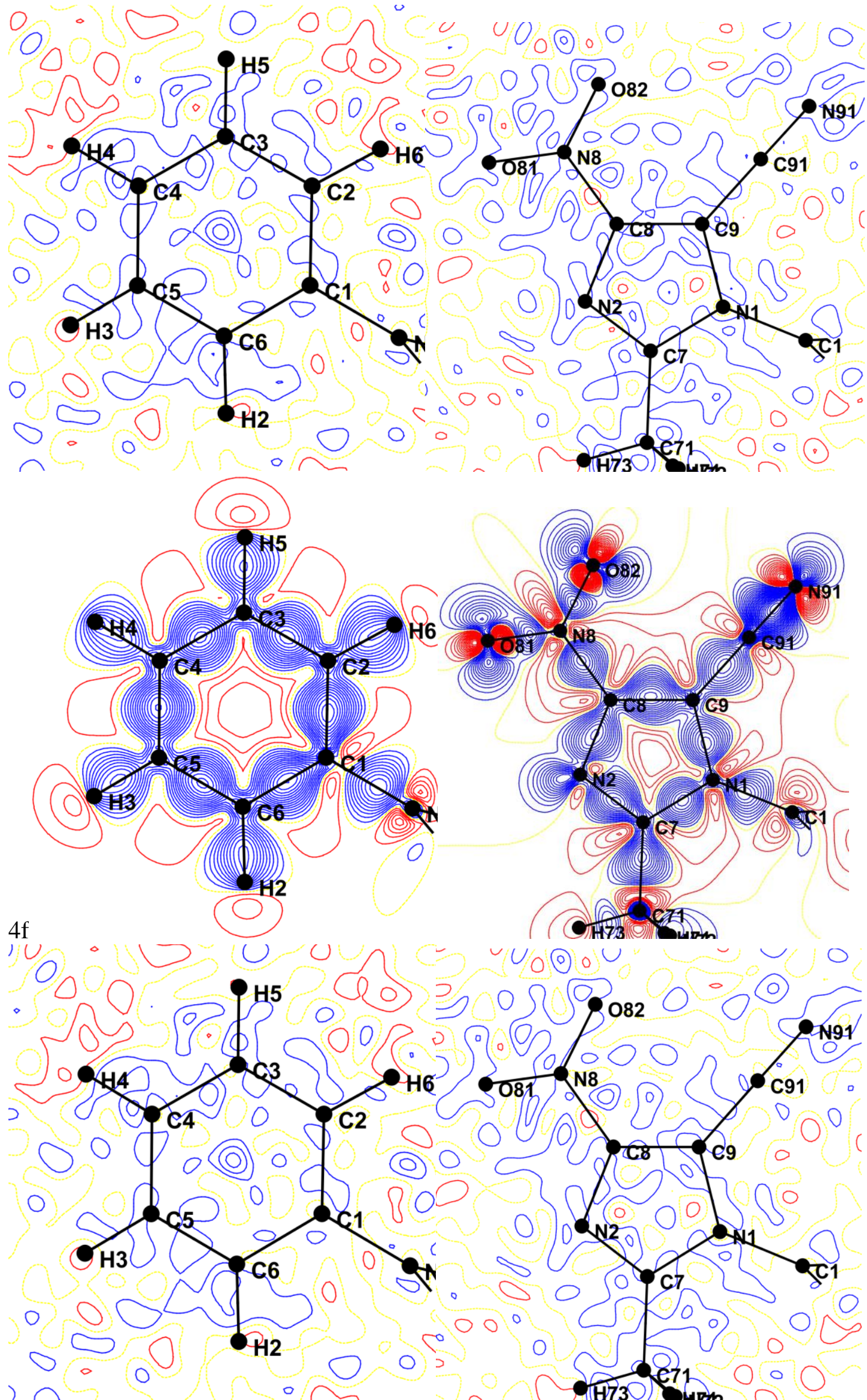

Figure S9. Fourier residual density maps for sub-models 4 not included in the main article, contour 0.05 , resolution $1 \mathrm{~A}^{-1}$ 